\title{
CURVED SQUEEZING OF UNBOUNDED DOMAINS AND TAIL ESTIMATES
}

\author{
KRZYSZTOF P. RYBAKOWSKI
}

This paper is dedicated to Professor Jean Mawhin on the occasion on his 70th birthday

\begin{abstract}
Using a resolvent convergence result from [7] we prove Conley index and index braid continuation results for reaction-diffusion equations on singularly perturbed unbounded curved squeezed domains
\end{abstract}

\section{Introduction}

Let $\omega$ be an arbitrary domain in $\mathbb{R}^{\ell}$, bounded or not, with Lipschitz boundary. We consider the following semilinear parabolic Neumann boundary problem

$$
\begin{aligned}
\widetilde{u}_{t} & =\Delta_{\omega} \widetilde{u}+\widetilde{G}_{\omega}(\widetilde{x}, \widetilde{u}), & & t>0, \widetilde{x} \in \omega, \\
\partial_{\nu_{\omega}} u & =0, & & t>0, \widetilde{x} \in \partial \omega,
\end{aligned}
$$

on $\omega$. Here, $\nu_{\omega}$ is the outer normal vector field to $\partial \omega$ and $\widetilde{G}_{\omega}: \omega \times \mathbb{R} \rightarrow \mathbb{R}$ is a suitable nonlinearity. Define the bilinear forms $\widetilde{a}_{\omega}$ and $\widetilde{b}_{\omega}$ by

$$
\begin{aligned}
& \widetilde{a}_{\omega}: H^{1}(\omega) \times H^{1}(\omega) \rightarrow \mathbb{R}, \quad(\widetilde{u}, \widetilde{v}) \mapsto \int_{\omega} \nabla \widetilde{u}(x) \cdot \nabla \widetilde{v}(x) d x ; \\
& \widetilde{b}_{\omega}: L^{2}(\omega) \times L^{2}(\omega) \rightarrow \mathbb{R}, \quad(\widetilde{u}, \widetilde{v}) \mapsto \int_{\omega} \widetilde{u}(x) \widetilde{v}(x) d x .
\end{aligned}
$$

Then the pair $\left(\widetilde{a}_{\omega}, \widetilde{b}_{\omega}\right)$ generates a densely defined selfadjoint operator $B_{\omega}$ on $L^{2}(\omega)$, which is commonly interpreted as the Laplace operator $-\Delta_{\omega}$ on $\omega$ with Neumann boundary condition.

2010 Mathematics Subject Classification. Primary 35B25, 37B30; Secondary 35B40, 35K57. Key words and phrases. reaction-diffusion equations, unbounded domains, Conley index. 
We are interested in the case $\omega=\Omega_{\varepsilon}$, where $\Omega_{\varepsilon}$, for $\varepsilon>0$ small, is 'thin' of order $\varepsilon$. As $\varepsilon \rightarrow 0^{+}$, the domain $\Omega_{\varepsilon}$ 'degenerates' to some limit set, which may no longer be a domain in $\mathbb{R}^{\ell}$.

More specifically, let $\mathcal{M} \subset \mathbb{R}^{\ell}$ be a smooth $\mathbf{k}$-dimensional submanifold of $\mathbb{R}^{\ell}$ and $\mathcal{U} \supset \mathcal{M}$ be a normal (tubular) neighbourhood of $\mathcal{M}$ with normal projection $\phi$. For $\varepsilon \in[0,1]$ define the squeezing operator $\Gamma_{\varepsilon}: \mathcal{U} \rightarrow \mathcal{U}$ by $x \mapsto \varepsilon x+(1-\varepsilon) \phi(x)$. For any domain $\Omega$ in $\mathbb{R}^{\ell}$ with Lipschitz boundary and $\mathrm{Cl} \Omega \subset \mathcal{U}$ we set $\Omega_{\varepsilon}=$ $\Gamma_{\varepsilon}(\Omega)$ and $\mathbf{B}_{\varepsilon}=B_{\Omega_{\varepsilon}}$ for $\left.\left.\varepsilon \in\right] 0,1\right]$. A particular case is the flat squeezing case in which, writing $\mathbb{R}^{\ell}=\mathbb{R}^{\mathbf{k}} \times \mathbb{R}^{\ell-\mathbf{k}}, x=\left(x_{1}, x_{2}\right)$, we set $\mathcal{M}=\mathbb{R}^{\mathbf{k}} \times\{0\}, \mathcal{U}=\mathbb{R}^{\ell}$ and $\phi(x)=\left(x_{1}, 0\right)$.

We also consider a family $G_{\varepsilon}: \mathcal{U} \times \mathbb{R} \rightarrow \mathbb{R}$ of suitable nonlinearities, $\varepsilon \in[0,1]$ and, for $\varepsilon \in] 0,1]$, set $\widetilde{G}_{\Omega_{\varepsilon}}:=G_{\varepsilon} \mid \Omega_{\varepsilon} \times \mathbb{R}$.

Equation (1.1) can be written abstractly as

$$
\dot{\tilde{u}}=-\mathbf{B}_{\varepsilon} \widetilde{u}+\widetilde{f}_{\varepsilon}(\widetilde{u})
$$

on $H^{1}\left(\Omega_{\varepsilon}\right)$ where $\widetilde{f}_{\varepsilon}$ is the Nemitski operator from $H^{1}\left(\Omega_{\varepsilon}\right)$ to $L^{2}\left(\Omega_{\varepsilon}\right)$ defined by $\widetilde{G}_{\Omega_{\varepsilon}}: \Omega_{\varepsilon} \times \mathbb{R} \rightarrow \mathbb{R}$.

Now using the change of variables defined by $\Gamma_{\varepsilon}$ we may pull $\mathbf{B}_{\varepsilon}$ back to $L^{2}(\Omega)$ and thus obtain the densely defined selfadjoint operator $\mathbf{A}_{\varepsilon}$ in $L^{2}(\Omega)$ given by:

(a) $\widetilde{u} \in D\left(\mathbf{B}_{\varepsilon}\right)$ if and only if $u=\widetilde{u} \circ \Gamma_{\varepsilon} \in D\left(\mathbf{A}_{\varepsilon}\right)$;

(b) $\mathbf{A}_{\varepsilon}(u)=\left(\mathbf{B}_{\varepsilon} \widetilde{u}\right) \circ \Gamma_{\varepsilon}$ for $\widetilde{u} \in D\left(\mathbf{B}_{\varepsilon}\right)$.

Equation (1.2) can then be pulled back to yield the equation

$$
\dot{u}=-\mathbf{A}_{\varepsilon} u+f_{\varepsilon}(u)
$$

on $H^{1}(\Omega)$. Here, $f_{\varepsilon}: H^{1}(\Omega) \rightarrow L^{2}(\Omega)$ is defined by

$$
f_{\varepsilon}\left(\widetilde{u} \circ \Gamma_{\varepsilon}\right)=\widetilde{f}_{\varepsilon}(\widetilde{u}) \circ \Gamma_{\varepsilon}, \quad \widetilde{u} \in H^{1}\left(\Omega_{\varepsilon}\right) .
$$

More explicitly,

$$
f_{\varepsilon}(u)(x)=G_{\varepsilon}\left(\Gamma_{\varepsilon}(x), u(x)\right)
$$

for $u \in H^{1}(\Omega)$ and $x \in \Omega$.

If $\Omega$ is bounded, then there is a closed linear subspace $L_{s}^{2}(\Omega)$ of $L^{2}(\Omega)$ and a densely defined selfadjoint operator $\mathbf{A}_{0}$ on $L_{s}^{2}(\Omega)$ such that, as $\varepsilon \rightarrow 0^{+}$, the eigenvalues and eigenfunctions of $\mathbf{A}_{\varepsilon}$ converge, in some sense, to the eigenvalues and eigenfunctions of $\mathbf{A}_{0}$, cf. [10], [13].

This spectral convergence theorem implies various Trotter-Kato-like linear convergence theorems of the $C^{0}$-semigroups $e^{-t \mathbf{A}_{\varepsilon}}$ to $e^{-t \mathbf{A}_{0}}$, cf. [10], [2], [13], which are used to prove attractor semicontinuity and Conley index continuation results for reaction-diffusion equations with nonlinearities satisfying certain growth assumptions, cf. [10], [13], [2], [3], [16]. 
If $\Omega$ is unbounded, then, in general, the operators $\mathbf{A}_{\varepsilon}$ do not have compact resolvents and so spectral convergence results in the above form are not expected to hold. However, as shown in [1] in the flat squeezing case, there is again a closed linear subspace $L_{s}^{2}(\Omega)$ of $L^{2}(\Omega)$ and a densely defined selfadjoint operator $\mathbf{A}_{0}$ on $L_{s}^{2}(\Omega)$ such that the resolvents of $\mathbf{A}_{\varepsilon}$ converge in some sense to the resolvents of $\mathbf{A}_{0}$. It turns out that this is sufficient for the validity of a corresponding linear convergence result.

In the recent paper [7] the above results from [1] were extended to the curved squeezing case provided the manifold $\mathcal{M}$ has bounded normal curvature. This condition is trivially satisfied in the flat squeezing case (the normal curvature of $\mathcal{M}=\mathbb{R}^{\mathbf{k}} \times\{0\}$ being zero) and for compact manifolds $\mathcal{M}$. A simple example of a noncompact manifold with bounded normal curvature is provided by the graph of the exponential function exp: $\mathbb{R} \rightarrow \mathbb{R}$, while the graph of the function $g:] 0, \infty[\rightarrow \mathbb{R}, x \mapsto \sin (1 / x)$, is a manifold with unbounded normal curvature.

Using the results from [7] we will obtain in this paper Conley index continuation results for problem (1.3) as $\varepsilon \rightarrow 0$.

This paper is organized as follows.

In Section 2 we introduce an abstract linear convergence concept (Lin) and relate it to resolvent convergence concept from [7]. We then define a nonlinear convergence concept (Conv) and establish some abstract nonlinear singular convergence results. Finally, we define the concept of singular $H^{\varepsilon}$-admissibility and show that this seemingly weaker concept actually implies the usual singular admissibility as defined in [4]. This implies various Conley index continuation results.

In Section 3 we will apply these abstract results to reaction-diffusion equations on singularly perturbed unbounded domains.

Some results on attractors on singularly perturbed unbounded domains are contained in [17].

\section{An abstract singular convergence result}

Definition 2.1. We say that the family $\left(H^{\varepsilon},\langle\cdot, \cdot\rangle_{H^{\varepsilon}}, A_{\varepsilon}\right)_{\varepsilon \in[0, \bar{\varepsilon}]}$ satisfies condition (Lin) if the following properties are satisfied:

(a) $\bar{\varepsilon} \in] 0, \infty\left[\right.$ and for every $\varepsilon \in[0, \bar{\varepsilon}],\left(H^{\varepsilon},\langle\cdot, \cdot\rangle_{H^{\varepsilon}}\right)$ is a Hilbert space and $A_{\varepsilon}: D\left(A_{\varepsilon}\right) \subset H^{\varepsilon} \rightarrow H^{\varepsilon}$ is a densely defined nonnegative self-adjoint operator on $\left(H^{\varepsilon},\langle\cdot, \cdot\rangle_{H^{\varepsilon}}\right)$. For $\alpha \in\left[0, \infty\left[\right.\right.$ write $H_{\alpha}^{\varepsilon}:=D\left(\left(A_{\varepsilon}+I_{\varepsilon}\right)^{\alpha / 2}\right)$, where $I_{\varepsilon}=\operatorname{Id}_{H^{\varepsilon}}$, and $\langle\cdot, \cdot\rangle_{H_{\alpha}^{\varepsilon}}:=\langle\cdot, \cdot\rangle_{\left(A_{\varepsilon}+I_{\varepsilon}\right)^{\alpha / 2}}$ with the corresponding norm $|\cdot|_{H_{\alpha}^{\varepsilon}}$. In particular, $H_{0}^{\varepsilon}=H^{\varepsilon}$;

(b) for each $\varepsilon \in] 0, \bar{\varepsilon}], H^{0}$ is a linear subspace of $H^{\varepsilon}$ and $H_{1}^{0}$ is a linear subspace of $H_{1}^{\varepsilon}$; 
(c) there exists a constant $C \in] 1, \infty[$ such that, for $\varepsilon \in] 0, \bar{\varepsilon}]$,

$$
|u|_{H_{1}^{\varepsilon}} \leq C|u|_{H_{1}^{0}} \quad \text { and } \quad|u|_{H_{1}^{0}} \leq C|u|_{H_{1}^{\varepsilon}}, \quad \text { whenever } u \in H_{1}^{0} ;
$$

(d) If $u_{0} \in H^{0}$ and $\left(u_{n}\right)_{n}$ is a sequence such that $u_{n} \in H^{\varepsilon_{n}}$ for each $n$ and $\left|u_{n}-u_{0}\right|_{H^{\varepsilon_{n}}} \rightarrow 0$ as $n \rightarrow \infty$, and if $\left(\varepsilon_{n}\right)_{n}$ is a sequence in $\left.] 0, \bar{\varepsilon}\right]$ with $\varepsilon_{n} \rightarrow 0$ then

$$
\left|e^{-t A_{\varepsilon_{n}}} u_{n}-e^{-t A_{0}} u_{0}\right|_{H_{1}^{\varepsilon_{n}}} \rightarrow 0 \text { as } n \rightarrow \infty,
$$

uniformly on compact intervals in $] 0, \infty[$.

(e) If $u_{0} \in H_{1}^{0}$ and $\left(u_{n}\right)_{n}$ is a sequence such that $u_{n} \in H_{1}^{\varepsilon_{n}}$ for each $n$ and $\left|u_{n}-u_{0}\right|_{H_{1}^{\varepsilon_{n}}} \rightarrow 0$ as $n \rightarrow \infty$, and if $\left(\varepsilon_{n}\right)_{n}$ is a sequence in $\left.] 0, \bar{\varepsilon}\right]$ with $\varepsilon_{n} \rightarrow 0$ then

$$
\left|e^{-t A_{\varepsilon_{n}}} u_{n}-e^{-t A_{0}} u_{0}\right|_{H_{1}^{\varepsilon_{n}}} \rightarrow 0 \text { as } n \rightarrow \infty,
$$

uniformly on compact intervals in $[0, \infty[$.

Proposition 2.2. If $\left(H^{\varepsilon},\langle\cdot, \cdot\rangle_{H^{\varepsilon}}, A_{\varepsilon}\right)_{\varepsilon \in[0, \bar{\varepsilon}]}$ satisfy condition (Lin), then for every $\varepsilon \in] 0, \bar{\varepsilon}]$, the subspace $H_{1}^{0}$ is closed in $\left(H_{1}^{\varepsilon},|\cdot|_{H_{1}^{\varepsilon}}\right)$. Let $Q_{\varepsilon}: H_{1}^{\varepsilon} \rightarrow H_{1}^{\varepsilon}$ be the $H_{1}^{\varepsilon}$-orthogonal projection of $H_{1}^{\varepsilon}$ onto $H_{1}^{0}$.

Definition 2.3. We say that the family $\left(H^{\varepsilon},\langle\cdot, \cdot\rangle_{H^{\varepsilon}}, A_{\varepsilon}\right)_{\varepsilon \in[0, \bar{\varepsilon}]}$ satisfies condition (Res) if the following properties are satisfied:

(a) $\bar{\varepsilon} \in] 0, \infty\left[\right.$ and for every $\varepsilon \in[0, \bar{\varepsilon}],\left(H^{\varepsilon},\langle\cdot, \cdot\rangle_{H^{\varepsilon}}\right)$ is a Hilbert space and $A_{\varepsilon}: D\left(A_{\varepsilon}\right) \subset H^{\varepsilon} \rightarrow H^{\varepsilon}$ is a densely defined nonnegative self-adjoint operator on $\left(H^{\varepsilon},\langle\cdot, \cdot\rangle_{H^{\varepsilon}}\right)$. For $\alpha \in\left[0, \infty\left[\right.\right.$ write $H_{\alpha}^{\varepsilon}:=D\left(\left(A_{\varepsilon}+I_{\varepsilon}\right)^{\alpha / 2}\right)$, where $I_{\varepsilon}=\operatorname{Id}_{H^{\varepsilon}}$, and $\langle\cdot, \cdot\rangle_{H_{\alpha}^{\varepsilon}}:=\langle\cdot, \cdot\rangle_{\left(A_{\varepsilon}+I_{\varepsilon}\right)^{\alpha / 2}}$ with the corresponding norm $|\cdot|_{H_{\alpha}^{\varepsilon}}$. In particular, $H_{0}^{\varepsilon}=H^{\varepsilon}$;

(b) for each $\varepsilon \in] 0, \bar{\varepsilon}], H^{0}$ is a linear subspace of $H^{\varepsilon}$ and $H_{1}^{0}$ is a linear subspace of $H_{1}^{\varepsilon}$;

(c) there exists a constant $C \in] 1, \infty[$ such that, for $\varepsilon \in] 0, \bar{\varepsilon}]$,

$$
|u|_{H_{1}^{\varepsilon}} \leq C|u|_{H_{1}^{0}} \quad \text { and } \quad|u|_{H_{1}^{0}} \leq C|u|_{H_{1}^{\varepsilon}}, \quad \text { whenever } u \in H_{1}^{0} \text {; }
$$

and

$$
|u|_{H^{\varepsilon}} \leq C|u|_{H^{0}} \quad \text { and } \quad|u|_{H^{0}} \leq C|u|_{H^{\varepsilon}}, \quad \text { whenever } u \in H^{0} ;
$$

(d) whenever $\left(\varepsilon_{n}\right)_{n}$ is a sequence in $\left.] 0, \bar{\varepsilon}\right]$ converging to zero, $w \in H^{0}$ and $\left(w_{n}\right)_{n}$ is a sequence in $H^{0}$ with $\left|w_{n}-w\right|_{H^{0}} \rightarrow 0$ as $n \rightarrow \infty$, then $\left|\left(A_{\varepsilon_{n}}+I_{\varepsilon_{n}}\right)^{-1} w_{n}-\left(A_{0}+I_{0}\right)^{-1} w\right|_{H_{1}^{\varepsilon_{n}}} \rightarrow 0$ as $n \rightarrow \infty$.

The following result is a rewording of [7, Theorem 3.4]. 
TheOrem 2.4. A family $\left(H^{\varepsilon},\langle\cdot, \cdot\rangle_{H^{\varepsilon}}, A_{\varepsilon}\right)_{\varepsilon \in[0, \bar{\varepsilon}]}$ satisfying condition (Res) satisfies condition (Lin).

Definition 2.5. Let $\bar{\varepsilon} \in] 0, \infty\left[\right.$ be arbitrary and $\left(H^{\varepsilon},\langle\cdot, \cdot\rangle_{H^{\varepsilon}}, A_{\varepsilon}\right)_{\varepsilon \in[0, \bar{\varepsilon}]}$ be a family satisfying condition (Lin). We say that the family $\left(f_{\varepsilon}\right)_{\varepsilon \in[0, \bar{\varepsilon}]}$ of maps satisfies condition (Conv) if the following properties are satisfied:

(a) $f_{\varepsilon}: H_{1}^{\varepsilon} \rightarrow H^{\varepsilon}$ for every $\varepsilon \in[0, \bar{\varepsilon}]$.

(b) $\lim _{\varepsilon \rightarrow 0^{+}}\left|e^{-t A_{\varepsilon}} f_{\varepsilon}(u)-e^{-t A_{0}} f_{0}(u)\right|_{H_{1}^{\varepsilon}}=0$ for every $u \in H_{1}^{0}$ and every $t \in$ ] $0, \infty[$.

(c) For every $M \in\left[0, \infty\left[\right.\right.$ there is an $L=L_{M} \in[0, \infty[$ such that

$$
\left|f_{\varepsilon}(u)-f_{\varepsilon}(v)\right|_{H^{\varepsilon}} \leq L|u-v|_{H_{1}^{\varepsilon}}
$$

for all $\varepsilon \in[0, \bar{\varepsilon}]$ and $u, v \in H_{1}^{\varepsilon}$ satisfying $|u|_{H_{1}^{\varepsilon}},|v|_{H_{1}^{\varepsilon}} \leq M$.

(d) For every $u \in H_{1}^{0}$ there is an $\left.\left.\varepsilon_{0}^{\prime} \in\right] 0, \bar{\varepsilon}\right]$ such that

$$
\sup _{\varepsilon \in\left[0, \varepsilon_{0}^{\prime}\right]}\left|f_{\varepsilon}(u)\right|_{H^{\varepsilon}}<\infty \text {. }
$$

REMARK 2.6. Note that, for $\alpha, t \in] 0, \infty[$ and $\lambda \in[0, \infty[$

$$
\lambda^{\alpha} e^{-\lambda t} \leq C(\alpha) t^{-\alpha} \quad \text { with } C(\alpha)=(\alpha / e)^{\alpha} .
$$

Let $\left(H^{\varepsilon},\langle\cdot, \cdot\rangle_{H^{\varepsilon}}, A_{\varepsilon}\right)_{\varepsilon \in[0, \bar{\varepsilon}]}$ satisfy condition (Lin). Let $\varepsilon \in[0, \bar{\varepsilon}]$ and $\left.r \in\right] 0, \infty[$. Using the Stone-Neumann operational calculus together with the above estimate with $\alpha=1 / 2$ we obtain the estimates

$$
\left|e^{-A_{\varepsilon} r} u\right|_{H^{\varepsilon}} \leq|u|_{H^{\varepsilon}}, \quad u \in H^{\varepsilon}
$$

and

$$
\left|e^{-A_{\varepsilon} r} u\right|_{H_{1}^{\varepsilon}} \leq C_{0} r^{-1 / 2} e^{r}|u|_{H^{\varepsilon}}, \quad u \in H^{\varepsilon}
$$

where $C_{0}=C(1 / 2)$.

The next result shows that the above condition (b) is valid uniformly for $t$ lying in compact subsets of $] 0, \infty[$.

Proposition 2.7. Assume condition (Conv) and let $\beta, \gamma \in] 0, \infty]$ be arbitrary with $\beta<\gamma$. Then, for every $u \in H_{1}^{0}$,

$$
\lim _{\varepsilon \rightarrow 0^{+}} \sup _{t \in[\beta, \gamma]}\left|e^{-t A_{\varepsilon}} f_{\varepsilon}(u)-e^{-t A_{0}} f_{0}(u)\right|_{H_{1}^{\varepsilon}}=0
$$

Proof. Let $v=e^{-\beta A_{0}} f_{0}(u) \in H_{1}^{0}$. For every $t \in[\beta, \gamma]$ we have

$$
\begin{aligned}
& \left|e^{-t A_{\varepsilon}} f_{\varepsilon}(u)-e^{-t A_{0}} f_{0}(u)\right|_{H_{1}^{\varepsilon}} \\
& \leq\left|e^{-(t-\beta) A_{\varepsilon}}\left(e^{-\beta A_{\varepsilon}} f_{\varepsilon}(u)-e^{-\beta A_{0}} f_{0}(u)\right)\right|_{H_{1}^{\varepsilon}}+\left|e^{-(t-\beta) A_{\varepsilon}} v-e^{-(t-\beta) A_{0}} v\right|_{H_{1}^{\varepsilon}} \\
& \leq\left|e^{-\beta A_{\varepsilon}} f_{\varepsilon}(u)-e^{-\beta A_{0}} f_{0}(u)\right|_{H_{1}^{\varepsilon}}+\left|e^{-(t-\beta) A_{\varepsilon}} v-e^{-(t-\beta) A_{0}} v\right|_{H_{1}^{\varepsilon}}
\end{aligned}
$$


Here we have used (2.1). Since, by part (e) of condition (Lin)

$$
\lim _{\varepsilon \rightarrow 0} \sup _{s \in[0, \gamma-\beta]}\left|e^{-s A_{\varepsilon}} v-e^{-s A_{0}} v\right|_{H_{1}^{\varepsilon}}=0,
$$

the assertion follows from condition (Conv) part (b) (with $t=\beta$ ).

For the rest of the paper, if $\left(H^{\varepsilon},\langle\cdot, \cdot\rangle_{H^{\varepsilon}}, A_{\varepsilon}\right)_{\varepsilon \in[0, \bar{\varepsilon}]}$ satisfies condition (Lin) and $\left(f_{\varepsilon}\right)_{\varepsilon \in[0, \bar{\varepsilon}]}$ satisfies condition (Conv) then we will write, for every $\varepsilon \in[0, \bar{\varepsilon}]$, $\pi_{\varepsilon}:=\pi_{A_{\varepsilon}, f_{\varepsilon}}$ to denote the local semiflow on $H_{1}^{\varepsilon}$ generated by the abstract parabolic equation

$$
\dot{u}=-A_{\varepsilon} u+f_{\varepsilon}(u) .
$$

Lemma 2.8. Suppose that $\left(H^{\varepsilon},\langle\cdot, \cdot\rangle_{H^{\varepsilon}}, A_{\varepsilon}\right)_{\varepsilon \in[0, \bar{\varepsilon}]}$ satisfies condition (Lin) and $\left(f_{\varepsilon}\right)_{\varepsilon \in[0, \bar{\varepsilon}]}$ satisfies condition (Conv). Let $\left.\left.\varepsilon_{0}^{\prime} \in\right] 0, \bar{\varepsilon}\right]$ be such that

$$
\sup _{\varepsilon \in\left[0, \varepsilon_{0}^{\prime}\right]}\left|f_{\varepsilon}(0)\right|_{H^{\varepsilon}}<\infty .
$$

( $\varepsilon_{0}^{\prime}$ exists in view of condition (Conv).) For every $\left.R \in\right] 0, \infty[$ there exists a $\tau=$ $\left.\tau_{R} \in\right] 0, \infty\left[\right.$ such that for every $\varepsilon \in\left[0, \varepsilon_{0}^{\prime}\right]$ and every $a \in H_{1}^{\varepsilon}$ with $|a|_{H_{1}^{\varepsilon}} \leq R, a \pi_{\varepsilon} t$ is defined and $\left|a \pi_{\varepsilon} t\right|_{H_{1}^{\varepsilon}} \leq 4 R$ for all $t \in[0, \tau]$.

Proof. Let $C$ be as in part (c) of condition (Lin), set

$$
M^{\prime}:=4 R
$$

and let $L:=L_{M^{\prime}}$ be as in Condition (Conv) with $M$ replaced by $M^{\prime}$. Set

$$
C_{1}=3 R \quad \text { and } \quad C_{2}=\sup _{\varepsilon \in\left[0, \varepsilon_{0}^{\prime}\right]}\left|f_{\varepsilon}(0)\right|_{H^{\varepsilon}}<\infty .
$$

Now choose $\tau \in] 0, \infty[$ with

$$
\begin{gathered}
2 C_{0} L \tau^{1 / 2} e^{\tau} \leq 1 / 2, \\
2 C_{0} \tau^{1 / 2} e^{\tau}\left(2 L C_{1}+C_{2}\right) \leq C_{1} / 4,
\end{gathered}
$$

where the constant $C_{0}$ is as in Remark 2.6. For every $\varepsilon \in\left[0, \varepsilon_{0}^{\prime}\right]$ and $a \in H_{1}^{\varepsilon}$ with

$$
|a|_{H_{1}^{\varepsilon}} \leq R
$$

define $S_{\varepsilon, a}:=\left\{u \mid u:[0, \tau] \rightarrow H_{1}^{\varepsilon}\right.$ is continuous and $|u(t)-a|_{H_{1}^{\varepsilon}} \leq C_{1}$ for all $t \in[0, \tau]\}$.

For $u \in S_{\varepsilon, a}$ define the map $T_{\varepsilon, a}(u):[0, \tau] \rightarrow H_{1}^{\varepsilon}$ by

$$
T_{\varepsilon, a}(u)(t):=e^{-t A_{\varepsilon}} a+\int_{0}^{t} e^{-(t-s) A_{\varepsilon}} f_{\varepsilon}(u(s)) d s .
$$

The map $T_{\varepsilon, a}(u)$ is continuous. Moreover, by (2.4), whenever $u \in S_{\varepsilon, a}$, then for all $t \in[0, \tau]$

$$
|u(t)|_{H_{1}^{\varepsilon}} \leq C_{1}+|a|_{H_{1}^{\varepsilon}} \leq 4 R=M^{\prime}
$$


Thus for all $u, v \in S_{\varepsilon, a}$ and for all $t \in[0, \tau]$, we have, by (2.2) and (2.6),

$$
\begin{aligned}
\mid T_{\varepsilon, a}(u)(t) & -\left.T_{\varepsilon, a}(v)(t)\right|_{H_{1}^{\varepsilon}} \\
= & \left|\int_{0}^{t} e^{-(t-s) A_{\varepsilon}}\left(f_{\varepsilon}(u(s))-f_{\varepsilon}(v(s))\right) d s\right|_{H_{1}^{\varepsilon}} \\
& \leq C_{0} \int_{0}^{t}(t-s)^{-1 / 2} e^{t-s}\left|f_{\varepsilon}(u(s))-f_{\varepsilon}(v(s))\right|_{H^{\varepsilon}} d s \\
& \leq C_{0} L e^{\tau} \int_{0}^{t}(t-s)^{-1 / 2} d s \sup _{s \in[0, \tau]}|u(s)-v(s)|_{H_{1}^{\varepsilon}} \\
& =2 C_{0} L \tau^{1 / 2} e^{\tau} \sup _{s \in[0, \tau]}|u(s)-v(s)|_{H_{1}^{\varepsilon}} \\
& \leq 1 / 2 \sup _{s \in[0, \tau]}|u(s)-v(s)|_{H_{1}^{\varepsilon}} .
\end{aligned}
$$

Moreover, for all $u \in S_{\varepsilon, a}$ and $t \in[0, \tau]$,

$$
\left|T_{\varepsilon, a}(u)(t)-a\right|_{H_{1}^{\varepsilon}} \leq\left|e^{-t A_{\varepsilon}} a-a\right|_{H_{1}^{\varepsilon}}+\left|\int_{0}^{t} e^{-(t-s) A_{\varepsilon}} f_{\varepsilon}(u(s)) d s\right|_{H_{1}^{\varepsilon}} .
$$

Since for $\varepsilon \in\left[0, \varepsilon_{0}^{\prime}\right]$ and $s \in[0, \tau]$ we have

$$
\begin{aligned}
\left|f_{\varepsilon}(u(s))\right|_{H^{\varepsilon}} & \leq\left|f_{\varepsilon}(u(s))-f_{\varepsilon}(a)\right|_{H^{\varepsilon}}+\left|f_{\varepsilon}(a)\right|_{H^{\varepsilon}} \\
& \leq L|u(s)-a|_{H_{1}^{\varepsilon}}+\left|f_{\varepsilon}(a)-f_{\varepsilon}(0)\right|_{H^{\varepsilon}}+\left|f_{\varepsilon}(0)\right|_{H^{\varepsilon}} \\
& \leq L C_{1}+L C_{1}+C_{2}=2 L C_{1}+C_{2},
\end{aligned}
$$

we obtain, by (2.7),

$$
\begin{aligned}
\left|\int_{0}^{t} e^{-(t-s) A_{\varepsilon}} f_{\varepsilon}(u(s)) d s\right|_{H_{1}^{\varepsilon}} & \leq C_{0} \int_{0}^{t}(t-s)^{-1 / 2} e^{(t-s)}\left|f_{\varepsilon}(u(s))\right|_{H^{\varepsilon}} d s \\
& \leq 2 C_{0} \tau^{1 / 2} e^{\tau}\left(2 L C_{1}+C_{2}\right) \leq C_{1} / 4=3 R / 4 .
\end{aligned}
$$

Moreover, $\left|e^{-t A_{\varepsilon}} a-a\right|_{H_{1}^{\varepsilon}} \leq\left|e^{-t A_{\varepsilon}} a\right|_{H_{1}^{\varepsilon}}+|a|_{H_{1}^{\varepsilon}} \leq|a|_{H_{1}^{\varepsilon}}+|a|_{H_{1}^{\varepsilon}} \leq 2 R$ by (2.1).

Altogether, we obtain

$$
\left|T_{\varepsilon, a}(u)(t)-a\right|_{H_{1}^{0}} \leq 2 R+3 R / 4 \leq 3 R=C_{1} \quad \text { for all } t \in[0, \tau] .
$$

Hence we conclude that $T_{\varepsilon, a}\left(S_{\varepsilon, a}\right) \subset S_{\varepsilon, a}$ and so, by (2.9) and Banach Fixed Point Theorem, there is a unique fixed point of $T_{\varepsilon, a}$ in $S_{\varepsilon, a}$. In particular $a \pi_{\varepsilon} t$ and is defined and $\left|a \pi_{\varepsilon} t\right|_{H_{1}^{\varepsilon}} \leq 4 R$ for all $t \in[0, \tau]$. The lemma is proved.

We can now state our first singular convergence result for semiflows.

Theorem 2.9. Suppose that the family $\left(H^{\varepsilon},\langle\cdot, \cdot\rangle_{H^{\varepsilon}}, A_{\varepsilon}\right)_{\varepsilon \in[0, \bar{\varepsilon}]}$ satisfies condition (Lin), and the family $\left(f_{\varepsilon}\right)_{\varepsilon \in[0, \bar{\varepsilon}]}$ satisfies condition (Conv). Let $\varepsilon_{0} \in[0, \bar{\varepsilon}]$ and $\left(\varepsilon_{n}\right)_{n}$ be a sequence in $[0, \bar{\varepsilon}]$ with $\varepsilon_{n} \rightarrow \varepsilon_{0}$. We assume that either $\varepsilon_{n}=\varepsilon_{0}$ 
for all $n \in \mathbb{N}$ or else $\varepsilon_{0}=0$ and $\varepsilon_{n}>0$ for all $n \in \mathbb{N}$. Let $u_{0} \in H_{1}^{\varepsilon_{0}}$ and $\left(u_{n}\right)_{n}$ be a sequence with $u_{n} \in H_{1}^{\varepsilon_{n}}$ for every $n \in \mathbb{N}$ and

$$
\left|u_{n}-u_{0}\right|_{H^{\varepsilon_{n}}} \rightarrow 0 \text { as } n \rightarrow \infty .
$$

Let $b \in] 0, \infty\left[\right.$ and suppose that $u_{n} \pi_{\varepsilon_{n}}$ t and $u_{0} \pi_{\varepsilon_{0}}$ t are defined for all $n \in \mathbb{N}$ and $t \in[0, b]$. Moreover, suppose there exists an $M^{\prime} \in\left[0, \infty\left[\right.\right.$ such that $\left|u_{n} \pi_{\varepsilon_{n}} s\right|_{H_{1}^{\varepsilon_{n}}} \leq$ $M^{\prime}$ for all $n \in \mathbb{N}$ and for all $s \in[0, b]$. Then for every $\left.\left.t \in\right] 0, b\right]$ and every sequence $\left(t_{n}\right)_{n}$ in $\left.] 0, b\right]$ converging to $t$

$$
\left|u_{n} \pi_{\varepsilon_{n}} t_{n}-u_{0} \pi_{\varepsilon_{0}} t_{n}\right|_{H_{1}^{\varepsilon_{n}}} \rightarrow 0 \quad \text { as } n \rightarrow \infty .
$$

Proof. Notice that $\widetilde{M}:=\sup _{s \in[0, b]}\left|u_{\varepsilon_{0}} \pi_{\varepsilon_{0}} s\right|_{H_{1}^{0}}<\infty$. Hence $\left|u_{\varepsilon_{0}} \pi_{\varepsilon_{0}} s\right|_{H_{1}^{\varepsilon_{n}}} \leq$ $C \widetilde{M}$ for all $s \in[0, b]$. Set $M^{\prime \prime}:=\max \left\{M^{\prime}, C \widetilde{M}\right\}$ and let $L:=L_{M^{\prime \prime}}$ be as in condition (Conv) with $M$ replaced by $M^{\prime \prime}$.

By the variation-of-constants formula we have, for all $n \in \mathbb{N}$ and all $t \in[0, b]$,

$$
\begin{aligned}
u_{n} \pi_{\varepsilon_{n}} t & -u_{0} \pi_{\varepsilon_{0}} t=e^{-t A_{\varepsilon_{n}}} u_{n}-e^{-t A_{\varepsilon_{0}}} u_{0} \\
& +\int_{0}^{t} e^{-(t-s) A_{\varepsilon_{n}}}\left(f_{\varepsilon_{n}}\left(u_{n} \pi_{\varepsilon_{n}} s\right)-f_{\varepsilon_{n}}\left(u_{0} \pi_{\varepsilon_{0}} s\right)\right) d s \\
& +\int_{0}^{t}\left(e^{-(t-s) A_{\varepsilon_{n}}} f_{\varepsilon_{n}}\left(u_{0} \pi_{\varepsilon_{0}} s\right)-e^{-(t-s) A_{\varepsilon_{0}}} f_{\varepsilon_{0}}\left(u_{0} \pi_{\varepsilon_{0}} s\right)\right) d s .
\end{aligned}
$$

Define the function $g_{n}:[0, b] \times[0, b] \rightarrow \mathbb{R}$ as follows: If $0<s<t$ then set

$$
g_{n}(t, s)=\left|e^{-(t-s) A_{\varepsilon_{n}}} f_{\varepsilon_{n}}\left(u_{0} \pi_{\varepsilon_{0}} s\right)-e^{-(t-s) A_{\varepsilon_{0}}} f_{\varepsilon_{0}}\left(u_{0} \pi_{\varepsilon_{0}} s\right)\right|_{H_{1}^{\varepsilon_{n}}}
$$

and set $g_{n}(t, s)=0$ otherwise. The function $g_{n}$ restricted to the set of $(s, t)$ with $0<s<t$ is continuous. Thus $g_{n}$ is measurable on $[0, b] \times[0, b]$. By Fubini's theorem the function

$$
c_{n}(t):=\int_{0}^{b} g_{n}(t, s) d s=\int_{0}^{t} g_{n}(t, s) d s
$$

is almost everywhere defined and measurable on $[0, b]$. Moreover, we obtain for $0<s<t$

$$
\left|g_{n}(t, s)\right| \leq C_{2} C_{0} e^{b}(t-s)^{-1 / 2}+C C_{2} C_{0} e^{b}(t-s)^{-1 / 2}=: C_{3}(t-s)^{-1 / 2},
$$

where

$$
C_{2}:=\max \left\{\sup _{s \in[0, b]} \sup _{n \in \mathbb{N}}\left|f_{\varepsilon_{n}}\left(u_{0} \pi_{0} s\right)\right|_{H^{\varepsilon_{n}}}, \sup _{s \in[0, b]}\left|f_{\varepsilon_{0}}\left(u_{0} \pi_{\varepsilon_{0}} s\right)\right|_{H^{0}}\right\} .
$$

Notice that condition (Conv) implies that $C_{2}<\infty$. Let $\left(t_{n}\right)_{n}$ be any sequence in $] 0, b]$ converging to some $t \in] 0, b]$. If $0<s<t$ then, then $0<s<t_{n}$ for all large $n$ and so Proposition 2.7 implies that $g_{n}\left(t_{n}, s\right) \rightarrow 0$ as $n \rightarrow \infty$. If $0<t<s$, then $0<t_{n}<s$ for all large $n$ and so again $g_{n}\left(t_{n}, s\right) \rightarrow 0$ as $n \rightarrow \infty$. It follows 
from (2.11) and the dominated convergence theorem that $c_{n}\left(t_{n}\right) \rightarrow 0$ as $n \rightarrow \infty$. Now (2.10) implies for all $r \in] 0, b]$

$$
\begin{aligned}
\left|u_{n} \pi_{\varepsilon_{n}} r-u_{0} \pi_{\varepsilon_{0}} r\right|_{H_{1}^{\varepsilon_{n}}} \leq & \left|e^{-r A_{\varepsilon_{n}}} u_{n}-e^{-r A_{\varepsilon_{0}}} u_{0}\right|_{H_{1}^{\varepsilon_{n}}}+c_{n}(r) \\
& +C_{0} e^{b} L_{M^{\prime \prime}} \int_{0}^{r}(r-s)^{-1 / 2}\left|u_{n} \pi_{\varepsilon_{n}} s-u_{0} \pi_{\varepsilon_{0}} s\right|_{H_{1}^{\varepsilon_{n}}} d s .
\end{aligned}
$$

For $n \in \mathbb{N}$ and $r \in] 0, b]$ set

$$
a_{n}(r)=\left|e^{-r A_{\varepsilon_{n}}} u_{n}-e^{-r A_{\varepsilon_{0}}} u_{0}\right|_{H_{1}^{\varepsilon_{n}}}+c_{n}(r) .
$$

It follows that $a_{n}$ is measurable and bounded on $\left.] 0, b\right]$. Using condition (Lin) we obtain that

$$
a_{n}\left(t_{n}\right) \rightarrow 0 \text { as } n \rightarrow \infty \text {. }
$$

An application of Henry's Inequality [8, Lemma 7.1.1] now implies that

$$
\left|u_{n} \pi_{\varepsilon_{n}} t_{n}-u_{0} \pi_{\varepsilon_{0}} t_{n}\right|_{H_{1}^{\varepsilon_{n}}} \leq a_{n}\left(t_{n}\right)+\int_{0}^{t_{n}} \rho\left(t_{n}-s\right) a_{n}(s) d s,
$$

where

$$
\rho(x):=\sum_{n=1}^{\infty} \frac{\left(C_{0} e^{b} L_{M^{\prime \prime}} \Gamma(\beta)\right)^{n}}{\Gamma(n \beta)} x^{n \beta-1}
$$

with $\beta:=1 / 2$.

The function $\rho:] 0, \infty[\rightarrow] 0, \infty[$ is well defined and continuous on $] 0, \infty[$ and it satisfies the estimate

$$
\left.\left.\rho(x) \leq C_{4} x^{-1 / 2}+C_{4} \quad \text { for } x \in\right] 0, b\right] .
$$

Fix a $\left.\delta_{0} \in\right] 0, t[$ and let $\delta \in] 0, \delta_{0} / 2\left[\right.$ be arbitrary. There is an $n_{0}=n_{0}(\delta) \in \mathbb{N}$ such that $\left|t_{n}-t\right|<\delta$ for $n \geq n_{0}$. Therefore for all such $n \in \mathbb{N}$ and all $s \in[0, t-2 \delta]$ it follows that $t_{n}-s>\delta$ so $\rho\left(t_{n}-s\right) \leq C_{4} \delta^{-1 / 2}+C_{4}$. Thus

$$
\left.\left.\rho\left(t_{n}-s\right) a_{n}(s) \leq C_{5} \quad \text { for } s \in\right] 0, t-2 \delta\right] .
$$

Therefore (2.12) and the dominated convergence theorem show that

$$
\int_{0}^{t-2 \delta} \rho\left(t_{n}-s\right) a_{n}(s) d s \rightarrow 0 \quad \text { as } n \rightarrow \infty .
$$

On the other hand,

$$
\int_{t-2 \delta}^{t_{n}} \rho\left(t_{n}-s\right) a_{n}(s) d s \leq C_{6}\left(\delta^{1 / 2}+\delta\right) .
$$

Since $\delta \in] 0, \delta_{0} / 2[$ is arbitrary, it follows that

$$
\int_{0}^{t_{n}} \rho\left(t_{n}-s\right) a_{n}(s) d s \rightarrow 0 \quad \text { as } n \rightarrow \infty .
$$

Consequently, $\left|u_{n} \pi_{\varepsilon_{n}} t_{n}-u_{0} \pi_{\varepsilon_{0}} t_{n}\right|_{H_{1}^{\varepsilon_{n}}} \rightarrow 0$ as $n \rightarrow \infty$. 
Lemma 2.10. Suppose that the family $\left(H^{\varepsilon},\langle\cdot, \cdot\rangle_{H^{\varepsilon}}, A_{\varepsilon}\right)_{\varepsilon \in[0, \bar{\varepsilon}]}$ satisfies condition (Lin) and the family $\left(f_{\varepsilon}\right)_{\varepsilon \in[0, \bar{\varepsilon}]}$ satisfies condition (Conv). Then for every $\widetilde{R} \in] 0, \infty\left[\right.$ there is a $\left.\widetilde{\tau}=\widetilde{\tau}_{\widetilde{R}} \in\right] 0, \infty\left[\right.$ such that whenever $u_{0} \in H_{1}^{0}$ is such that $\left|u_{0}\right|_{H_{1}^{0}} \leq \widetilde{R},\left(\varepsilon_{n}\right)_{n}$ is a sequence in $\left.] 0, \bar{\varepsilon}\right]$ with $\varepsilon_{n} \rightarrow 0$ and $\left(u_{n}\right)_{n}$ is a sequence with $u_{n} \in H_{1}^{\varepsilon_{n}}$ for every $n \in \mathbb{N}$ and

$$
\left|u_{n}-u_{0}\right|_{H_{1}^{\varepsilon_{n}}} \rightarrow 0 \text { as } n \rightarrow \infty,
$$

then there exist an $n_{0} \in \mathbb{N}$ such that $u_{0} \pi_{0} t$ and $u_{n} \pi_{\varepsilon_{n}} t$ are defined for all $n \geq n_{0}$ and $t \in[0, \widetilde{\tau}]$ and

$$
\sup _{t \in[0, \widetilde{\tau}]}\left|u_{n} \pi_{\varepsilon_{n}} t-u_{0} \pi_{0} t\right|_{H_{1}^{\varepsilon_{n}}} \rightarrow 0 \quad \text { as } n \rightarrow \infty .
$$

Proof. Let $\varepsilon_{0}^{\prime}$ be as in Lemma 2.8 and $\left.\widetilde{R} \in\right] 0, \infty[$ be arbitrary. Define $R=(1+C) \widetilde{R}$ and $\widetilde{\tau}=\tau_{R}$, where $\tau_{R}$ be as in Lemma 2.8. Let $u_{0} \in H_{1}^{0}$ be arbitrary such that $\left|u_{0}\right|_{H_{1}^{0}} \leq \widetilde{R}$. Moreover, let $\left(\varepsilon_{n}\right)_{n}$ be a sequence in $\left.] 0, \bar{\varepsilon}\right]$ with $\varepsilon_{n} \rightarrow 0$ and $\left(u_{n}\right)_{n}$ be a sequence with $u_{n} \in H_{1}^{\varepsilon_{n}}$ for every $n \in \mathbb{N}$ and such that $\left|u_{n}-u_{0}\right|_{H_{1}^{\varepsilon_{n}}} \rightarrow 0$ as $n \rightarrow \infty$. Then there is an $n_{0} \in \mathbb{N}$ such that, $\varepsilon_{n} \leq \varepsilon_{0}^{\prime}$ and $\left|u_{n}-u_{0}\right|_{H_{1}^{\varepsilon_{n}}} \leq \widetilde{R}$ for $n \geq n_{0}$. Thus, for all such $n$

$$
\left|u_{n}\right|_{H_{1}^{\varepsilon_{n}}} \leq\left|u_{n}-u_{0}\right|_{H_{1}^{\varepsilon_{n}}}+\left|u_{0}\right|_{H_{1}^{\varepsilon_{n}}} \leq\left|u_{n}-u_{0}\right|_{H_{1}^{\varepsilon_{n}}}+C\left|u_{0}\right|_{H_{1}^{0}} \leq R .
$$

If follows from Lemma 2.8 that $u_{0} \pi_{0} s$ and $u_{n} \pi_{\varepsilon_{n}} s$ are defined for $n \geq n_{0}$ and $s \in[0, \widetilde{\tau}]$ and $\left|u_{n} \pi_{0} s\right|_{H_{1}^{0}} \leq C_{1}$ and $\left|u_{n} \pi_{\varepsilon_{n}} s\right|_{H_{1}^{\varepsilon_{n}}} \leq C_{1}$ for $n \geq n_{0}$ and $s \in[0, \widetilde{\tau}]$. Here, $C_{1}=4 R$.

If the lemma does not hold then, taking subsequences if necessary, we may assume that there is a sequence $\left(t_{n}\right)_{n \in \mathbb{N}}$ in $[0, \widetilde{\tau}]$ converging to some $t_{0} \in[0, \widetilde{\tau}]$ and there is a $\delta \in] 0, \infty[$ such that

$$
\left|u_{n} \pi_{\varepsilon_{n}} t_{n}-u_{0} \pi_{0} t_{n}\right|_{H_{1}^{\varepsilon_{n}}} \geq \delta, \quad n \geq n_{0} .
$$

If $t_{0}>0$, then (2.13) contradicts Theorem 2.9. Therefore $t_{0}=0$. For every $t \in[0, \tau]$ we have

$$
u_{n} \pi_{\varepsilon_{n}} t-u_{0}=e^{-t A_{\varepsilon_{n}}} u_{n}-u_{0}+\int_{0}^{t} e^{-(t-s) A_{\varepsilon_{n}}} f_{\varepsilon_{n}}\left(u_{n} \pi_{\varepsilon_{n}} s\right) d s .
$$

Note that $\left|u_{0} \pi_{0} s\right|_{H_{1}^{\varepsilon_{n}}} \leq C C_{1}$ for all $s \in[0, \tau]$. Let $L:=L_{M}$ be as in (Conv) with $M=C C_{1}$. It follows that for all $n \geq n_{0}$ and for every $s \in[0, \tau]$

$$
\begin{aligned}
\left|f_{\varepsilon_{n}}\left(u_{n} \pi_{\varepsilon_{n}} s\right)\right|_{H^{\varepsilon_{n}}} & \leq\left|f_{\varepsilon_{n}}\left(u_{n} \pi_{\varepsilon_{n}} s\right)-f_{\varepsilon_{n}}\left(u_{0}\right)\right|_{H^{\varepsilon_{n}}}+\left|f_{\varepsilon_{n}}\left(u_{0}\right)\right|_{H^{\varepsilon_{n}}} \\
& \leq L\left|u_{n} \pi_{\varepsilon_{n}} s-u_{0}\right|_{H_{1}^{\varepsilon_{n}}}+\left|f_{\varepsilon_{n}}\left(u_{0}\right)\right|_{H^{\varepsilon_{n}}} \\
& \leq L\left(C_{1}+C\left|u_{0}\right|_{H_{1}^{0}}\right)+\left|f_{\varepsilon_{n}}\left(u_{0}\right)\right|_{H^{\varepsilon_{n}}} .
\end{aligned}
$$

Part (d) of condition (Conv) now implies

$$
\left|f_{\varepsilon_{n}}\left(u_{n} \pi_{\varepsilon_{n}} s\right)\right|_{H^{\varepsilon_{n}}} \leq \widetilde{C}, \text { for all } s \in[0, \tau] \text { and for all } n \geq n_{0},
$$


for some $\widetilde{C} \in] 0, \infty\left[\right.$. Therefore for all $n \geq n_{0}$

$$
\begin{aligned}
\mid u_{n} \pi_{\varepsilon_{n}} t_{n} & -\left.u_{0} \pi_{0} t_{n}\right|_{H_{1}^{\varepsilon_{n}}} \leq\left|u_{n} \pi_{\varepsilon_{n}} t_{n}-u_{0}\right|_{H_{1}^{\varepsilon_{n}}}+C\left|u_{0} \pi_{0} t_{n}-u_{0}\right|_{H_{1}^{0}} \\
& \leq\left|e^{-t_{n} A_{\varepsilon_{n}}} u_{n}-e^{-t_{n} A_{0}} u_{0}\right|_{H_{1}^{\varepsilon_{n}}}+C\left|e^{-t_{n} A_{0}} u_{0}-u_{0}\right|_{H_{1}^{0}} \\
& +C_{0} \widetilde{C} \int_{0}^{t_{n}}\left(t_{n}-s\right)^{-1 / 2} d s+C\left|u_{0} \pi_{0} t_{n}-u_{0}\right|_{H_{1}^{0}} .
\end{aligned}
$$

Since $\left|e^{-t_{n} A_{0}} u_{0}-u_{0}\right|_{H_{1}^{0}} \rightarrow 0$ and $\left|u_{0} \pi_{0} t_{n}-u_{0}\right|_{H_{1}^{0}} \rightarrow 0$ as $n \rightarrow \infty$, condition (Lin) implies that $\left|u_{n} \pi_{\varepsilon_{n}} t_{n}-u_{0} \pi_{0} t_{n}\right|_{H_{1}^{\varepsilon_{n}}} \rightarrow 0$ as $n \rightarrow \infty$, but this contradicts (2.13). The lemma is proved.

We conclude this section proving our main convergence result for semiflows.

TheOREM 2.11. Suppose the family $\left(H^{\varepsilon},\langle\cdot, \cdot\rangle_{H^{\varepsilon}}, A_{\varepsilon}\right)_{\varepsilon \in[0, \bar{\varepsilon}]}$ satisfies condition (Lin) and the family $\left(f_{\varepsilon}\right)_{\varepsilon \in[0, \bar{\varepsilon}]}$ satisfy condition (Conv). Let $\left(\varepsilon_{n}\right)_{n}$ be a sequence in $] 0, \bar{\varepsilon}]$ with $\varepsilon_{n} \rightarrow 0$ and let $\left(t_{n}\right)_{n}$ be a sequence in $\left[0, \infty\left[\right.\right.$ with $t_{n} \rightarrow t_{0}$, for some $t_{0} \in\left[0, \infty\left[\right.\right.$. Let $u_{0} \in H_{1}^{0}$ and $\left(u_{n}\right)_{n}$ be a sequence with $u_{n} \in H_{1}^{\varepsilon_{n}}$ for every $n \in \mathbb{N}$ and

$$
\left|u_{n}-u_{0}\right|_{H_{1}^{\varepsilon_{n}}} \rightarrow 0 \text { as } n \rightarrow \infty .
$$

Assume $u_{0} \pi_{0} t_{0}$ is defined. Then there exists an $n_{0} \in \mathbb{N}$ such that $u_{n} \pi_{\varepsilon_{n}} t_{n}$ is defined for all $n \geq n_{0}$ and

$$
\left|u_{n} \pi_{\varepsilon_{n}} t_{n}-u_{0} \pi_{0} t_{0}\right|_{H_{1}^{\varepsilon_{n}}} \rightarrow 0 \text { as } n \rightarrow \infty .
$$

Proof. Since $u_{0} \pi_{0} t_{0}$ is defined, there is a $\left.b>t_{0}, b \in\right] 0, \infty\left[\right.$, such that $u_{0} \pi_{0} t$ is defined for all $t \in[0, b[$. Define

$$
\begin{gathered}
I:=\left\{t \in \left[0, b\left[\mid \text { there exists an } n_{0} \in \mathbb{N} \text { such that } u_{n} \pi_{\varepsilon_{n}} t \text { is defined for } n \geq n_{0}\right.\right.\right. \\
\left.\quad \text { and } \sup _{s \in[0, t]}\left|u_{n} \pi_{\varepsilon_{n}} s-u_{0} \pi_{0} s\right|_{H_{1}^{\varepsilon_{n}}} \rightarrow 0 \text { as } n \rightarrow \infty\right\} .
\end{gathered}
$$

It is clear that $0 \in I$. Furthermore if $0 \leq t^{\prime}<t$ and $t \in I$, then $t^{\prime} \in I$. Let

$$
\bar{t}:=\sup I \text {. }
$$

It follows that $\bar{t} \leq b$ and so $[0, \bar{t}[\subset I$. An application of Lemma 2.10 with an arbitrary $\left.\widetilde{R}_{0} \in\right] 0, \infty\left[\right.$ with $\left|u_{0}\right|_{H_{1}^{0}} \leq \widetilde{R}_{0}$ and a corresponding $\widetilde{\tau}_{0}=\widetilde{\tau}_{\widetilde{R}_{0}}$ shows that $\bar{t}>\widetilde{\tau}_{0}>0$. We claim that $\bar{t}=b$. Suppose, on the contrary, that $\bar{t}<b$. It follows that $u_{0} \pi_{0} \bar{t}$ is defined. Let $\left.\widetilde{R}_{1} \in\right] 0, \infty$ [ be arbitrary with $\left|u_{0} \pi_{0} \bar{t}\right|_{H_{1}^{0}}<\widetilde{R}_{1}$ and $\widetilde{\tau}_{1}=\widetilde{\tau}_{\widetilde{R}_{1}}$ be as in Lemma 2.10 . By continuity of $\pi_{0}$ there is a $t \in \mathbb{R}$ with $0<t<\bar{t}<t+\widetilde{\tau}_{1}$ and $\left|u_{0} \pi_{0} t\right|_{H_{1}^{0}}<\widetilde{R}_{1}$. We have that $t \in I$ so there exists an $n_{0} \in \mathbb{N}$ such that $u_{n} \pi_{\varepsilon_{n}} t$ is defined for all $n \geq n_{0}$ and

$$
\sup _{s \in[0, t]}\left|u_{n} \pi_{\varepsilon_{n}} s-u_{0} \pi_{0} s\right|_{H_{1}^{\varepsilon_{n}}} \rightarrow 0 \quad \text { as } n \rightarrow \infty
$$


Lemma 2.10 implies that there is an $n_{1} \in \mathbb{N}$ with $n_{1} \geq n_{0}$ such that $\left(u_{0} \pi_{0} t\right) \pi_{0} s$ and $\left(u_{n} \pi_{\varepsilon_{n}} t\right) \pi_{\varepsilon_{n}} s$ are defined for all $n \geq n_{1}$ and $s \in\left[0, \widetilde{\tau}_{1}\right]$ and

$$
\sup _{s \in\left[0, \widetilde{\tau}_{1}\right]}\left|\left(u_{n} \pi_{\varepsilon_{n}} t\right) \pi_{\varepsilon_{n}} s-\left(u_{0} \pi_{0} t\right) \pi_{0} s\right|_{H_{1}^{\varepsilon_{n}}} \rightarrow 0 \quad \text { as } n \rightarrow \infty .
$$

Formulas (2.14) and (2.15) imply that $u_{0} \pi_{0}\left(t+\widetilde{\tau}_{1}\right)$ is defined, $u_{n} \pi_{\varepsilon_{n}}\left(t+\widetilde{\tau}_{1}\right)$ is also defined for all $n \geq n_{1}$ and

$$
\sup _{s \in\left[0, t+\widetilde{\tau}_{1}\right]}\left|u_{n} \pi_{\varepsilon_{n}} s-u_{0} \pi_{0} s\right|_{H_{1}^{\varepsilon_{n}}} \rightarrow 0 \quad \text { as } n \rightarrow \infty .
$$

Thus $t+\widetilde{\tau}_{1} \in I$, but $t+\widetilde{\tau}_{1}>\bar{t}$, a contradiction, which proves that $\bar{t}=b$.

Since $t_{0} \in\left[0, b\left[\right.\right.$, it follows that there is a $t \in\left[0, b\left[\right.\right.$ with $t_{0}<t$ and $t_{n}<t$ for all $n$ large enough. In particular $u_{0} \pi_{0} t_{n}$ and $u_{n} \pi_{\varepsilon_{n}} t_{n}$ are defined for all $n$ large enough and

$$
\left|u_{n} \pi_{\varepsilon_{n}} t_{n}-u_{0} \pi_{0} t_{n}\right|_{H_{1}^{\varepsilon_{n}}} \rightarrow 0 \quad \text { as } n \rightarrow \infty .
$$

Since

$$
\left|u_{0} \pi_{0} t_{n}-u_{0} \pi_{0} t_{0}\right|_{H_{1}^{\varepsilon_{n}}} \leq C\left|u_{0} \pi_{0} t_{n}-u_{0} \pi_{0} t_{0}\right|_{H_{1}^{0}}
$$

and $\left|u_{0} \pi_{0} t_{n}-u_{0} \pi_{0} t_{0}\right|_{H_{1}^{0}} \rightarrow 0$ as $n \rightarrow \infty$, the theorem follows.

Definition 2.12. Suppose that the family $\left(H^{\varepsilon},\langle\cdot, \cdot\rangle_{H^{\varepsilon}}, A_{\varepsilon}\right)_{\varepsilon \in[0, \bar{\varepsilon}]}$ satisfies condition (Lin) and the family $\left(f_{\varepsilon}\right)_{\varepsilon \in[0, \bar{\varepsilon}]}$ satisfies condition (Conv). Let $\beta \in$ ] $0, \infty\left[\right.$ and $N$ be a closed subset of $H_{1}^{0}$. For $\varepsilon \in[0, \bar{\varepsilon}]$ set

$$
\begin{array}{lr}
N_{\varepsilon, \beta}=N, & \text { if } \varepsilon=0, \\
N_{\varepsilon, \beta}=\left\{u \in H_{1}^{\varepsilon} \mid Q_{\varepsilon} u \in N \text { and }\left|\left(\operatorname{Id}_{H_{1}^{\varepsilon}}-Q_{\varepsilon}\right) u\right|_{H_{1}^{\varepsilon}} \leq \beta\right\}, & \text { if } \varepsilon>0 .
\end{array}
$$

We say that $N$ is strongly admissible rel. to $\beta$ and the family $\left(H_{1}^{\varepsilon}, \pi_{\varepsilon}\right)_{\varepsilon \in[0, \bar{\varepsilon}]}$, (resp. the family $\left(H^{\varepsilon}, \pi_{\varepsilon}\right)_{\varepsilon \in[0, \bar{\varepsilon}]}$ ) if the following conditions are satisfied:

(a) for each $\varepsilon \in[0, \bar{\varepsilon}]$ the local semiflow $\pi_{\varepsilon}$ does not explode in $N_{\varepsilon, \beta}$;

(b) whenever $\varepsilon_{0} \in[0, \bar{\varepsilon}],\left(\varepsilon_{n}\right)_{n}$ is a sequence in $[0, \bar{\varepsilon}],\left(t_{n}\right)_{n}$ is a sequence in ] $0, \infty\left[\right.$ and $\left(u_{n}\right)_{n}$ is a sequence such that

$$
t_{n} \rightarrow \infty \text { as } n \rightarrow \infty,
$$

either $\varepsilon_{n}=\varepsilon_{0}$ for all $n \in \mathbb{N}$ or else $\varepsilon_{0}=0$ and $\varepsilon_{n} \rightarrow \varepsilon_{0}$ for $n \rightarrow \infty$, and, for each $n \in \mathbb{N}, u_{n} \in H_{1}^{\varepsilon_{n}}$ and $u_{n} \pi_{\varepsilon_{n}}\left[0, t_{n}\right] \subset N_{\varepsilon_{n}, \beta}$, then there exist a $v \in H_{1}^{\varepsilon_{0}}$ and a subsequence of the sequence $\left(u_{n} \pi_{\varepsilon_{n}} t_{n}\right)_{n \in \mathbb{N}}$, denoted again by $\left(u_{n} \pi_{\varepsilon_{n}} t_{n}\right)_{n \in \mathbb{N}}$, such that

$$
\begin{array}{lll} 
& \left|u_{n} \pi_{\varepsilon_{n}} t_{n}-v\right|_{H_{1}^{\varepsilon_{n}}} \rightarrow 0 \quad \text { as } n \rightarrow \infty . \\
\text { (resp. } & \left.\left|u_{n} \pi_{\varepsilon_{n}} t_{n}-v\right|_{H^{\varepsilon_{n}}} \rightarrow 0 \quad \text { as } n \rightarrow \infty .\right)
\end{array}
$$

REMARK 2.13. If $N$ is strongly admissible rel. to $\beta$ and $\left(H_{1}^{\varepsilon}, \pi_{\varepsilon}\right)_{\varepsilon \in[0, \bar{\varepsilon}]}$ and $v$ is as in (b) of Definition 2.12, then, as is easily seen, $v \in N_{\varepsilon_{0}, \beta}$. 
Theorem 2.14. Suppose that the family $\left(H^{\varepsilon},\langle\cdot, \cdot\rangle_{H^{\varepsilon}}, A_{\varepsilon}\right)_{\varepsilon \in[0, \bar{\varepsilon}]}$ satisfies condition (Lin) and the family $\left(f_{\varepsilon}\right)_{\varepsilon \in[0, \bar{\varepsilon}]}$ satisfies condition (Conv). Let $\beta \in$ ] $0, \infty\left[\right.$ and $N$ be a closed bounded subset of $H_{1}^{0}$. Suppose $N$ is strongly admissible rel. to $\beta$ and the family $\left(H^{\varepsilon}, \pi_{\varepsilon}\right)_{\varepsilon \in[0, \bar{\varepsilon}]}$. Then $N$ is strongly admissible rel. to $\beta$ and the family $\left(H_{1}^{\varepsilon}, \pi_{\varepsilon}\right)_{\varepsilon \in[0, \bar{\varepsilon}]}$.

Proof. There is an $M \in\left[0, \infty\left[\right.\right.$ such that $|w|_{H_{1}^{0}} \leq M$ so $|w|_{H_{1}^{\varepsilon}} \leq C M$ for all $w \in N$.

Let $\varepsilon_{0} \in[0, \bar{\varepsilon}],\left(\varepsilon_{n}\right)_{n}$ be a sequence in $[0, \bar{\varepsilon}],\left(t_{n}\right)_{n}$ be a sequence in $] 0, \infty[$ and $\left(u_{n}\right)_{n}$ be a sequence such that

$$
t_{n} \rightarrow \infty \text { as } n \rightarrow \infty,
$$

either $\varepsilon_{n}=\varepsilon_{0}$ for all $n \in \mathbb{N}$ or else $\varepsilon_{0}=0$ and $\varepsilon_{n} \rightarrow \varepsilon_{0}$ for $n \rightarrow \infty$,

and, for each $n \in \mathbb{N}, u_{n} \in H_{1}^{\varepsilon_{n}}$ and $u_{n} \pi_{\varepsilon_{n}}\left[0, t_{n}\right] \subset N_{\varepsilon_{n}, \beta}$. There is an $n_{0} \in \mathbb{N}$ such that $t_{n} \geq 1$ for all $n \geq n_{0}$. Set $t_{n}^{\prime}=t_{n}-1$ for all $n \geq n_{0}$. By hypothesis there is a $v \in N_{\varepsilon_{0}, \beta}$ and a subsequence of the endpoint sequence $\left(u_{n} \pi_{\varepsilon_{n}} t_{n}^{\prime}\right)_{n \geq n_{0}}$, denoted again by $\left(u_{n} \pi_{\varepsilon_{n}} t_{n}^{\prime}\right)_{n \geq n_{0}}$, such that

$$
\left|u_{n} \pi_{\varepsilon_{n}} t_{n}^{\prime}-v\right|_{H^{\varepsilon_{n}}} \rightarrow 0 \text { as } n \rightarrow \infty .
$$

Let $v_{n}=u_{n} \pi_{\varepsilon_{n}} t_{n}^{\prime}, n \geq n_{0}$. Then, for all $n \geq n_{0}$ and $s \in[0,1], v_{n} \pi_{\varepsilon_{n}} s$ is defined and $v_{n} \pi_{\varepsilon_{n}} s \in N_{\varepsilon_{n}, \beta}$ so

$$
\left|v_{n} \pi_{\varepsilon_{n}} s\right|_{H_{1}^{\varepsilon_{n}}} \leq\left|Q_{\varepsilon_{n}}\left(v_{n} \pi_{\varepsilon_{n}} s\right)\right|_{H_{1}^{\varepsilon_{n}}}+\left|\left(\operatorname{Id}_{H_{1}^{\varepsilon}}-Q_{\varepsilon}\right)\left(v_{n} \pi_{\varepsilon_{n}} s\right)\right|_{H_{1}^{\varepsilon_{n}}} \leq C M+\beta,
$$

SO

$$
\left|v_{n} \pi_{\varepsilon_{n}} s\right|_{H_{1}^{\varepsilon_{n}}} \leq C M+\beta, \quad n \geq n_{0}, \quad s \in[0,1] .
$$

There is an $\left.s_{1} \in\right] 0,1\left[\right.$ such that $v \pi_{\varepsilon_{0}} s_{1}$ is defined. Therefore, the estimate (2.16) together with Theorem 2.9 show that

$$
\left|w_{n}-w\right|_{H_{1}^{\varepsilon_{n}}} \rightarrow 0, \quad \text { as } n \rightarrow \infty
$$

where $w=v \pi_{\varepsilon_{0}} s_{1}$ and $w_{n}=v_{n} \pi_{\varepsilon_{n}} s_{1}$ for $n \geq n_{0}$. If $\varepsilon_{n}=\varepsilon_{0}$ for all $n \geq n_{0}$, then this implies that $w \in N_{\varepsilon_{0}, \beta}$. If $\varepsilon_{0}=0$ and $\varepsilon_{n} \rightarrow \varepsilon_{0}$ as $n \rightarrow \infty$, then, as $Q_{\varepsilon_{n}} w_{n} \in N$ for all $n \geq n_{0}$ and $Q_{\varepsilon} w=w$ for all $\left.\left.\varepsilon \in\right] 0, \bar{\varepsilon}\right]$, we obtain from the estimates

$$
\left|Q_{\varepsilon_{n}} w_{n}-w\right|_{H_{1}^{0}}=\left|Q_{\varepsilon_{n}}\left(w_{n}-w\right)\right|_{H_{1}^{0}} \leq C\left|Q_{\varepsilon_{n}}\left(w_{n}-w\right)\right|_{H_{1}^{\varepsilon_{n}}} \leq C\left|w_{n}-w\right|_{H_{1}^{\varepsilon_{n}}}
$$

that $w \in N=N_{\varepsilon_{0}, \beta}$. Thus $w \in N_{\varepsilon_{0}, \beta}$ in both cases. We claim that $w \pi_{\varepsilon_{0}}\left(1-s_{1}\right)$ is defined. If not, then the fact that $\pi_{\varepsilon_{0}}$ does not explode in $N_{\varepsilon_{0}, \beta}$ implies that there is some $\left.s_{2} \in\right] 0,1-s_{1}\left[\right.$ such that $w \pi_{\varepsilon_{0}} s_{2}$ is defined and $w \pi_{\varepsilon_{0}} s_{2} \notin N_{\varepsilon_{0}, \beta}$. If $\varepsilon_{n}=\varepsilon_{0}$ for all $n \geq n_{0}$, then continuity of $\pi_{\varepsilon_{0}}$ implies that $w_{n} \pi_{\varepsilon_{n}} s_{2} \notin N_{\varepsilon_{n}, \beta}$, for all $n$ large enough, which contradicts the fact that $w_{n} \pi_{\varepsilon_{n}} s_{2}=v_{n} \pi_{\varepsilon_{n}}\left(s_{1}+s_{2}\right)$ for 
all $n \geq n_{0}$ and $s_{1}+s_{2}<1$. If $\varepsilon_{0}=0$ and $\varepsilon_{n} \rightarrow \varepsilon_{0}$ as $n \rightarrow \infty$, then an application of Theorem 2.11 shows that

$$
\left|w_{n}^{\prime}-w^{\prime}\right|_{H_{1}^{\varepsilon_{n}}} \rightarrow 0, \quad \text { as } n \rightarrow \infty
$$

where $w^{\prime}=w \pi_{\varepsilon_{0}} s_{2}$ and $w_{n}^{\prime}=w_{n} \pi_{\varepsilon_{n}} s_{2}$ for $n \geq n_{0}$. As above this implies that $w^{\prime} \in N_{\varepsilon_{0}, \beta}$, a contradiction. This proves that $w \pi_{\varepsilon_{0}}\left(1-s_{1}\right)$ is defined so $v \pi_{\varepsilon_{0}} s$ is defined for all $s \in[0,1]$. Thus the estimate (2.16) together with Theorem 2.9 show that

$$
\left|u_{n} \pi_{\varepsilon_{n}} t_{n}-v \pi_{\varepsilon_{0}} 1\right|_{H_{1}^{\varepsilon_{n}}}=\left|v_{n} \pi_{\varepsilon_{n}} 1-v \pi_{\varepsilon_{0}} 1\right|_{H_{1}^{\varepsilon_{n}}} \rightarrow 0, \quad \text { as } n \rightarrow \infty .
$$

The theorem is proved.

Let the family $\left(H^{\varepsilon},\langle\cdot, \cdot\rangle_{H^{\varepsilon}}, A_{\varepsilon}\right)_{\varepsilon \in[0, \bar{\varepsilon}]}$ satisfy condition (Lin) and the family $\left(f_{\varepsilon}\right)_{\varepsilon \in[0, \bar{\varepsilon}]}$ satisfy condition (Conv).

Set $X_{0}:=H_{1}^{0}$. For every $\left.\left.\varepsilon \in\right] 0, \varepsilon_{0}\right]$, define $Y_{\varepsilon}:=\left(I-Q_{\varepsilon}\right) H_{1}^{\varepsilon}$ and endow $Y_{\varepsilon}$ with the norm $|\cdot|_{H_{1}^{\varepsilon}}$ restricted to $Y_{\varepsilon}$. Define on $Z_{\varepsilon}=X_{0} \times Y_{\varepsilon}$ the following norm:

$$
\|(u, v)\|_{\varepsilon}:=\max \left\{|u|_{H_{1}^{0}},|v|_{H_{1}^{\varepsilon}}\right\} \quad \text { for }(u, v) \in Z_{\varepsilon} .
$$

We will denote by $\widetilde{\Gamma}_{\varepsilon}$ the metric on $Z_{\varepsilon}$ induced by the norm $\|\cdot\|_{\varepsilon}$. For each $\left.\varepsilon \in] 0, \varepsilon_{0}\right]$, define $\theta_{\varepsilon}:=0$.

Let $\Psi_{\varepsilon}: H_{1}^{\varepsilon} \rightarrow Z_{\varepsilon}$ be the linear map defined by

$$
\Psi_{\varepsilon}(w):=\left(Q_{\varepsilon} w,\left(I-Q_{\varepsilon}\right) w\right) \text { for } w \in H_{1}^{\varepsilon} .
$$

It follows that $\Psi_{\varepsilon}$ is a bijective linear map and its inverse map is given by

$$
\Psi_{\varepsilon}^{-1}(u, v)=u+v \quad \text { for }(u, v) \in Z_{\varepsilon} .
$$

Moreover, both $\Psi_{\varepsilon}$ and $\Psi_{\varepsilon}^{-1}$ are continuous maps. This fact is a consequence of the following inequalities:

$$
\begin{aligned}
\left\|\Psi_{\varepsilon}(w)\right\|_{\varepsilon} \leq C|w|_{H_{1}^{\varepsilon}} & & \text { for } w \in H_{1}^{\varepsilon}, \\
\left|\Psi_{\varepsilon}{ }^{-1}(u, v)\right|_{H_{1}^{\varepsilon}} \leq\left(1+C^{2}\right)^{1 / 2}\|(u, v)\|_{\varepsilon} & & \text { for }(u, v) \in Z_{\varepsilon},
\end{aligned}
$$

where the constant $C \in] 1, \infty[$ was defined in hypothesis (Lin).

Given $(u, v) \in Z_{\varepsilon}$ and $t \in[0, \infty[$ define

$$
(u, v) \tilde{\pi}_{\varepsilon} t:=\Psi_{\varepsilon}\left(\Psi_{\varepsilon}{ }^{-1}(u, v) \pi_{\varepsilon} t\right)
$$

whenever $\Psi_{\varepsilon}^{-1}(u, v) \pi_{\varepsilon} t$ is defined. It follows that $\tilde{\pi}_{\varepsilon}$ is a local semiflow on $Z_{\varepsilon}$, the conjugate to $\pi_{\varepsilon}$ via $\Psi_{\varepsilon}$. Theorem 2.11 and inequalities (2.17) and (2.18) immediately imply the following 
Corollary 2.15. Under the above hypotheses the family $\left(\tilde{\pi}_{\varepsilon}\right)_{\left.\varepsilon \in] 0, \varepsilon_{0}\right]}$ converges singularly to $\pi_{0}$.

Theorem 2.14, Remark 2.13 and inequalities (2.17) and (2.18) imply the following:

COROllary 2.16. Under the above hypotheses let $\beta \in] 0, \infty[$ and $N$ be a closed bounded subset of $H_{1}^{0}$. If $N$ is strongly admissible rel. to $\beta$ and the family $\left(H^{\varepsilon}, \pi_{\varepsilon}\right)_{\varepsilon \in[0, \bar{\varepsilon}]}$, then $N$ is singularly strongly admissible with respect to $\beta$ and the family $\left(\widetilde{\pi}_{\varepsilon}\right)_{\varepsilon \in[0, \bar{\varepsilon}]}$, where $\widetilde{\pi}_{0}=\pi_{0}$.

We can now state the following Conley index continuation principle for singular families of abstract parabolic equations:

THEOREM 2.17. Suppose that the family $\left(H^{\varepsilon},\langle\cdot, \cdot\rangle_{H^{\varepsilon}}, A_{\varepsilon}\right)_{\varepsilon \in[0, \bar{\varepsilon}]}$ satisfies condition (Lin) and the family $\left(f_{\varepsilon}\right)_{\varepsilon \in[0, \bar{\varepsilon}]}$ satisfies condition (Conv). Let $\beta \in$ ]0, $\infty$ [ and $N$ be a closed bounded subset of $H_{1}^{0}$. Suppose $N$ is strongly admissible rel. to $\beta$ and the family $\left(H^{\varepsilon}, \pi_{\varepsilon}\right)_{\varepsilon \in[0, \bar{\varepsilon}]}$. Finally, assume $N$ is an isolating neighbourhood of an invariant set $K_{0}$ relative to $\pi_{0}$. For $\left.\left.\varepsilon \in\right] 0, \varepsilon_{0}\right]$ and for every $\eta \in] 0, \infty[$ set

$$
N_{\varepsilon, \eta}:=\left\{u \in H_{1}^{\varepsilon} \mid Q_{\varepsilon} u \in N \text { and }\left|\left(I_{\varepsilon}-Q_{\varepsilon}\right) u\right|_{H_{1}^{\varepsilon}} \leq \eta\right\}
$$

and $K_{\varepsilon, \eta}:=\operatorname{Inv}_{\pi_{\varepsilon}}\left(N_{\varepsilon, \eta}\right)$ i.e. $K_{\varepsilon, \eta}$ is the largest $\pi_{\varepsilon}$-invariant set in $N_{\varepsilon, \eta}$. Then for every $\eta \in] 0, \beta]$ there exists an $\left.\left.\varepsilon^{\mathrm{c}}=\varepsilon^{\mathrm{c}}(\eta) \in\right] 0, \varepsilon_{0}\right]$ such that for every $\left.\left.\varepsilon \in\right] 0, \varepsilon^{\mathrm{c}}\right]$ the set $N_{\varepsilon, \eta}$ is a strongly admissible isolating neighbourhood of $K_{\varepsilon, \eta}$ relative to $\pi_{\varepsilon}$ and

$$
h\left(\pi_{\varepsilon}, K_{\varepsilon, \eta}\right)=h\left(\pi_{0}, K_{0}\right) .
$$

Furthermore, for every $\eta>0$, the family $\left(K_{\varepsilon, \eta}\right)_{\varepsilon \in\left[0, \varepsilon^{c}(\eta)\right]}$ of invariant sets, where $K_{0, \eta}=K_{0}$, is upper semicontinuous at $\varepsilon=0$ with respect to the family $|\cdot|_{H_{1}^{\varepsilon}}$ of norms i.e.

$$
\lim _{\varepsilon \rightarrow 0^{+}} \sup _{w \in K_{\varepsilon, \eta}} \inf _{u \in K_{0}}|w-u|_{H_{1}^{\varepsilon}}=0
$$

Proof. The isomorphism $\Psi_{\varepsilon}$ conjugates the local semiflow $\pi_{\varepsilon}$ to the local semiflow $\tilde{\pi}_{\varepsilon}$. Thus whenever $S$ is a strongly admissible isolating neighbourhood with respect to $\pi_{\varepsilon}$, then $\Psi_{\varepsilon}(S)$ is a strongly admissible isolating neighbourhood with respect to $\tilde{\pi}_{\varepsilon}$ and

$$
h\left(\pi_{\varepsilon}, S\right)=h\left(\tilde{\pi}_{\varepsilon}, \Psi_{\varepsilon}(S)\right) .
$$

Corollaries 2.15 and 2.16 imply that the family of semiflows $\left(\widetilde{\pi}_{\varepsilon}\right)_{\varepsilon \in\left[0, \varepsilon_{0}\right]}$ and the set $N$ satisfy the hypotheses of [4, Theorem 4.1]. Notice also that any closed ball in $Y_{\varepsilon}$ is contractible. Hence [4, Theorem 4.1] and [4, Corollary 4.11] completes the proof. 
REMARK. The family $\left(K_{\varepsilon, \eta}\right)_{\left.\varepsilon \in] 0, \varepsilon^{c}(\eta)\right]}$ is asymptotically independent of $\eta$ i.e. whenever $\eta_{1}$ and $\left.\eta_{2} \in\right] 0, \infty\left[\right.$ then there is an $\left.\left.\varepsilon^{\prime} \in\right] 0, \min \left(\varepsilon^{\mathrm{c}}\left(\eta_{1}\right), \varepsilon^{\mathrm{c}}\left(\eta_{2}\right)\right)\right]$ such that $K_{\varepsilon, \eta_{1}}=K_{\varepsilon, \eta_{2}}$ for $\left.\left.\varepsilon \in\right] 0, \varepsilon^{\prime}\right]$.

We also state the following (co)homology index continuation principle:

TheOREM 2.18. Assume the hypotheses of Theorem 2.17 and for every $\eta \in$ ] $0, \infty\left[\right.$ let $\left.\left.\varepsilon^{\mathrm{c}}(\eta) \in\right] 0, \varepsilon_{0}\right]$ be as in that theorem. Let $(P, \prec)$ be a finite poset. Let $\left(M_{p, 0}\right)_{p \in P}$ be a $\prec$-ordered Morse decomposition of $K_{0}$ relative to $\pi_{0}$. For each $p \in P$, let $V_{p} \subset N$ be closed in $X_{0}$ and such that $M_{p, 0}=\operatorname{Inv}_{\pi_{0}}\left(V_{p}\right) \subset \operatorname{Int}_{H_{1}^{0}}\left(V_{p}\right)$. (Such sets $V_{p}, p \in P$, exist.) For $\left.\left.\varepsilon \in\right] 0, \varepsilon_{0}\right]$, for every $\left.\eta \in\right] 0, \infty[$ and $p \in P$ set $M_{p, \varepsilon, \eta}:=\operatorname{Inv}_{\pi_{\varepsilon}}\left(V_{p, \varepsilon, \eta}\right)$, where

$$
V_{p, \varepsilon, \eta}:=\left\{u \in H_{1}^{\varepsilon} \mid Q_{\varepsilon} u \in V_{p} \text { and }\left|\left(I-Q_{\varepsilon}\right) u\right|_{H_{1}^{\varepsilon}} \leq \eta\right\} .
$$

Then for every $\eta \in] 0, \infty[$ there is an $\left.\widetilde{\varepsilon}=\widetilde{\varepsilon}(\eta) \in] 0, \varepsilon^{\mathrm{c}}(\eta)\right]$ such that for every $\varepsilon \in] 0, \widetilde{\varepsilon}]$ and $p \in P, M_{p, \varepsilon, \eta} \subset \operatorname{Int}_{H_{1}^{\varepsilon}}\left(V_{p, \varepsilon, \eta}\right)$ and the family $\left(M_{p, \varepsilon, \eta}\right)_{p \in P}$ is $a \prec$-ordered Morse decomposition of $K_{\varepsilon, \eta}$ relative to $\pi_{\varepsilon}$ and the (co)homology index braids of $\left(\pi_{0}, K_{0},\left(M_{p, 0}\right)_{p \in P}\right)$ and $\left.\left.\left.\left(\pi_{\varepsilon}, K_{\varepsilon, \eta},\left(M_{p, \varepsilon, \eta}\right)_{p \in P}\right)\right), \varepsilon \in\right] 0, \widetilde{\varepsilon}\right]$, are isomorphic and so they determine the same collection of $C$-connection matrices.

Proof. Since the isomorphism $\Psi_{\varepsilon}$ conjugates the local semiflow $\pi_{\varepsilon}$ to the local semiflow $\widetilde{\pi}_{\varepsilon}$, using [6, Proposition 2.7], it follows that whenever $S$ is a strongly admissible isolating neighbourhood with respect to $\pi_{\varepsilon}$ and $\left(M_{p}\right)_{p \in P}$ is a $\prec-$ ordered Morse decomposition of $S$ relative to $\pi_{\varepsilon}$, then $\Psi_{\varepsilon}(S)$ is a strongly admissible isolating neighbourhood with respect to $\widetilde{\pi}_{\varepsilon}$ and $\left(\Psi_{\varepsilon}\left(M_{p}\right)\right)_{p \in P}$ is a $\prec$-ordered Morse decomposition of $S$ relative to $\widetilde{\pi}_{\varepsilon}$ and the (co)homology index braids of $\left(\pi_{\varepsilon}, S,\left(M_{p}\right)_{p \in P}\right)$ and $\left.\left.\left.\left(\widetilde{\pi}_{\varepsilon}, \Psi_{\varepsilon}(S),\left(\Psi_{\varepsilon}\left(M_{p}\right)\right)_{p \in P}\right)\right), \varepsilon \in\right] 0, \varepsilon_{0}\right]$, are isomorphic.

Corollaries 2.15 and 2.16 imply that the family of semiflows $\left(\widetilde{\pi}_{\varepsilon}\right)_{\varepsilon \in\left[0, \varepsilon_{0}\right]}$ and the set $N$ satisfy the hypotheses of [5, Theorem 3.10]. Since any closed ball in $Y_{\varepsilon}$ is contractible, an application of [5, Theorem 3.10] completes the proof.

Remark. Again, for each $p \in P$, the family $\left(M_{p, \varepsilon, \eta}\right)_{\varepsilon \in[0, \widetilde{\varepsilon}(\eta)]}$, where $M_{p, 0, \eta}=$ $M_{p, 0}$ is upper semicontinuous at $\varepsilon=0$ with respect to the family $|\cdot|_{H_{1}^{\varepsilon}}$ of norms and the family $\left(M_{p, \varepsilon, \eta}\right)_{\varepsilon \in] 0, \widetilde{\varepsilon}(\eta)]}$ is asymptotically independent of $\eta$.

\section{Applications to curved squeezing on unbounded domains}

We will now apply the previous results to singularly perturbed equations on curvedly squeezed unbounded domains. We assume the reader's familiarity with the paper [7] and only recall some necessary definitions.

Let $\ell, \mathbf{k}$ and $\mathbf{r}$ be positive integers with $\mathbf{r} \geq 2, \ell \geq 2$ and $\mathbf{k}<\ell$. Let $\langle\cdot, \cdot\rangle$ be an inner product on $\mathbb{R}^{\ell}$ and $\|\cdot\|$ be the corresponding Euclidean norm.

Let $\mathcal{M} \subset \mathbb{R}^{\ell}$ be a $\mathbf{k}$-dimensional submanifold of $\mathbb{R}^{\ell}$ of class $C^{\mathbf{r}}$. 
For $p \in \mathcal{M}$ let $Q(p): \mathbb{R}^{\ell} \rightarrow \mathbb{R}^{\ell}$ be the orthogonal projection of $\mathbb{R}^{\ell}$ onto the tangent space $T_{p}(\mathcal{M})$ to $\mathcal{M}$ at $p$ and $P(p): \mathbb{R}^{\ell} \rightarrow \mathbb{R}^{\ell}$ be the orthogonal projection of $\mathbb{R}^{\ell}$ onto the orthogonal complement $T_{p}^{\perp}(\mathcal{M})$ of $T_{p}(\mathcal{M})$ in $\mathbb{R}^{\ell}$. We have $P(p)=\operatorname{Id}_{\mathbb{R}^{\ell}}-Q(p)$.

The map $Q: \mathcal{M} \rightarrow \mathcal{L}\left(\mathbb{R}^{\ell}, \mathbb{R}^{\ell}\right)$ is of class $C^{\mathbf{r}-1}$.

Moreover, $(D Q(p) a) b \in T_{p}^{\perp}(\mathcal{M})$ for each $p \in \mathcal{M}$ and all $a, b \in T_{p}(\mathcal{M})$ and the map

$$
\mathrm{II}_{p}: T_{p}(\mathcal{M}) \times T_{p}(\mathcal{M}) \rightarrow T_{p}^{\perp}(\mathcal{M}), \quad(a, b) \mapsto(D Q(p) a) b
$$

is bilinear and symmetric. The map $\mathrm{II}_{p}$ is called the second fundamental form of $\mathcal{M}$ at $p$.

We say that $\mathcal{M}$ has bounded second fundamental form if

$$
\sup \left\{\left\|\mathrm{II}_{p}(a, b)\right\| \mid p \in \mathcal{M},(a, b) \in T_{p}(\mathcal{M}) \times T_{p}(\mathcal{M}),\|a\| \leq 1,\|b\| \leq 1\right\}<\infty .
$$

This is equivalent to the requirement that

$\sup \left\{\|(D Q(p) a) c\| \mid p \in \mathcal{M},(a, c) \in T_{p}(\mathcal{M}) \times T_{p}^{\perp}(\mathcal{M}),\|a\| \leq 1,\|c\| \leq 1\right\}<\infty$.

Definition 3.1. An open set $\mathcal{U}$ in $\mathbb{R}^{\ell}$ with $\mathcal{M} \subset \mathcal{U}$ is called a normal neighbourhood (or normal strip) of $\mathcal{M}$ if there is a map $\phi: \mathcal{U} \rightarrow \mathcal{M}$ of class $C^{\mathbf{r}-1}$, called an orthogonal projection of $\mathcal{U}$ onto $\mathcal{M}$ and a continuous function $\delta: \mathcal{M} \rightarrow] 0, \infty]$, called the thickness of $\mathcal{U}$ such that:

(a) whenever $x \in \mathcal{U}$ and $p \in \mathcal{M}$ then $\phi(x)=p$ if and only if the vector $x-p \in T_{p}^{\perp} \mathcal{M}$ and $\|x-p\|<\delta(p)$;

(b) $\varepsilon x+(1-\varepsilon) \phi(x) \in \mathcal{U}$ for all $x \in \mathcal{U}$ and all $\varepsilon \in[0,1]$.

For the rest of this paper assume that $\mathcal{M}$ has bounded second fundamental form choose $M \in] 0, \infty[$ arbitrarily with

$\sup \left\{\|(D Q(p) a) c\| \mid(p, a, c) \in \mathcal{M} \times T_{p}(\mathcal{M}) \times T_{p}^{\perp}(\mathcal{M}),\|a\| \leq 1,\|c\| \leq 1\right\} \leq M$.

Proposition 3.2. Let $\left.q_{0} \in\right] 0,1[$ be arbitrary. There is a normal neighbourhood $\mathcal{U}$ of $\mathcal{M}$ with normal projection $\phi$ and thickness $\delta$ such that $M \delta(p) \leq q_{0}$ for all $p \in \mathcal{M}$.

For the rest of this paper we fix a $\left.q_{0} \in\right] 0,1[$ and a normal neighbourhood $\mathcal{U}$ with normal projection $\phi$ and thickness $\delta$ such that the assertions of Proposition 3.2 are satisfied.

For $\varepsilon \in[0,1]$ define the maps:

- $\Gamma_{\varepsilon}: \mathcal{U} \rightarrow \mathcal{U}$ by $x \mapsto \phi(x)+\varepsilon(x-\phi(x))$,

- $J_{\varepsilon}: \mathcal{U} \rightarrow \mathbb{R}$ by $J_{\varepsilon}(x)=\left|\operatorname{det}\left(D \Gamma_{\varepsilon}(x)_{\mid T_{\phi(x)}(\mathcal{M})}\right)\right|, x \in \mathcal{U}$, and

- $S_{\varepsilon}: \mathcal{U} \rightarrow \mathcal{L}\left(\mathbb{R}^{\ell}, \mathbb{R}^{\ell}\right)$ by

$$
S_{\varepsilon}(x) h=D \phi\left(\Gamma_{\varepsilon}(x)\right) h-\left(D Q(\phi(x))\left(D \phi\left(\Gamma_{\varepsilon}(x)\right) h\right)\right)(x-\phi(x))
$$




$$
\text { for } x \in \mathcal{U} \text { and } h \in \mathbb{R}^{\ell} \text {. }
$$

In the sequel, given a linear map $B: \mathbb{R}^{\ell} \rightarrow \mathbb{R}^{\ell}$ we denote by $B^{T}$ the adjoint of $B$ relative to the scalar product $\langle\cdot, \cdot\rangle$.

For the rest of this paper we will assume that

$$
\left.\left.\Omega \text { is open in } \mathbb{R}^{\ell} \text { with } \mathrm{Cl}(\Omega) \subset \mathcal{U} \text {. For } \varepsilon \in\right] 0,1\right] \text {, we write } \Omega_{\varepsilon}=\Gamma_{\varepsilon}(\Omega) .
$$

For $\varepsilon \in] 0,1]$ define the following bilinear forms:

$$
\begin{gathered}
\widetilde{a}_{\varepsilon}: H^{1}\left(\Omega_{\varepsilon}\right) \times H^{1}\left(\Omega_{\varepsilon}\right) \rightarrow \mathbb{R}, \quad(\widetilde{u}, \widetilde{v}) \mapsto \int_{\Omega_{\varepsilon}} \nabla \widetilde{u}(x) \cdot \nabla \widetilde{v}(x) d x ; \\
\widetilde{b}_{\varepsilon}: L^{2}\left(\Omega_{\varepsilon}\right) \times L^{2}\left(\Omega_{\varepsilon}\right) \rightarrow \mathbb{R}, \quad(\widetilde{u}, \widetilde{v}) \mapsto \int_{\Omega_{\varepsilon}} \widetilde{u}(x) \widetilde{v}(x) d x ;
\end{gathered}
$$

and let $a_{\varepsilon}: H^{1}(\Omega) \times H^{1}(\Omega) \rightarrow \mathbb{R}$ be defined by

$$
\begin{aligned}
a_{\varepsilon}(u, v)= & \int_{\Omega} J_{\varepsilon}(x)\left\langle S_{\varepsilon}(x)^{T} \nabla u(x), S_{\varepsilon}(x)^{T} \nabla v(x)\right\rangle d x \\
& +\frac{1}{\varepsilon^{2}} \int_{\Omega} J_{\varepsilon}(x)\langle P(x) \nabla u(x), P(x) \nabla v(x)\rangle d x, \quad u, v \in H^{1}(\Omega) .
\end{aligned}
$$

For $\varepsilon \in[0,1]$ define the bilinear form $b_{\varepsilon}: L^{2}(\Omega) \times L^{2}(\Omega) \rightarrow \mathbb{R}$ by

$$
b_{\varepsilon}(u, v)=\int_{\Omega} J_{\varepsilon}(x) u(x) v(x) d x, u, v \in L^{2}(\Omega) .
$$

We have

$$
\left.\left.\widetilde{a}_{\varepsilon}(u, u)+\widetilde{b}_{\varepsilon}(u, u)=|u|_{H^{1}\left(\Omega_{\varepsilon}\right)}^{2}, \quad \varepsilon \in\right] 0,1\right], u \in H^{1}\left(\Omega_{\varepsilon}\right) .
$$

Let $\varepsilon \in] 0,1]$ be arbitrary. Then the pair $\left(\widetilde{a}_{\varepsilon}, \widetilde{b}_{\varepsilon}\right)$ generates a densely defined selfadjoint operator $\mathbf{B}_{\varepsilon}$ in $\left(L^{2}\left(\Omega_{\varepsilon}\right), \widetilde{b}_{\varepsilon}\right)$, which we interpret, as usual, as the operator $-\Delta$ on $\Omega_{\varepsilon}$ with Neumann boundary condition on $\partial \Omega_{\varepsilon}$.

Let us define the space

$$
H_{s}^{1}(\Omega):=\left\{u \in H^{1}(\Omega) \mid P(x) \nabla u(x)=0 \text { a.e. }\right\} .
$$

Note that

$$
\begin{aligned}
& u \in H_{s}^{1}(\Omega) \text { iff } u \in H^{1}(\Omega) \text { and }\langle\nabla u(x), \nu\rangle \text { for a.a. } x \in \Omega \text { and all } \\
& \nu \in T_{\phi(x)}^{\perp}(\mathcal{M}) .
\end{aligned}
$$

This is a closed linear subspace of the Hilbert space $H^{1}(\Omega)$. Now define the 'limit' bilinear form

$$
a_{0}: H_{s}^{1}(\Omega) \times H_{s}^{1}(\Omega) \rightarrow \mathbb{R}, \quad(u, v) \mapsto \int_{\Omega} J_{0}(x)\left\langle S_{0}(x)^{T} \nabla u(x), S_{0}(x)^{T} \nabla v(x)\right\rangle d x .
$$

Finally, let $L_{s}^{2}(\Omega)$ be the closure of $H_{s}^{1}(\Omega)$ in $L^{2}(\Omega) . L_{s}^{2}(\Omega)$ is a closed linear subspace of the Hilbert space $L^{2}(\Omega)$. For $\left.\left.\varepsilon \in\right] 0,1\right]$ and $u, v \in L^{2}(\Omega)$ set

$$
\langle u, v\rangle_{\varepsilon}:=b_{\varepsilon}(u, v) \text {. }
$$


For $\varepsilon \in] 0,1]$ and $u, v \in H^{1}(\Omega)$ set

$$
\langle\langle u, v\rangle\rangle_{\varepsilon}:=a_{\varepsilon}(u, v)+b_{\varepsilon}(u, v) .
$$

$\langle\cdot, \cdot\rangle_{\varepsilon}\left(\right.$ resp. $\left\langle\langle\cdot, \cdot\rangle_{\varepsilon}\right)$ is a scalar product on $H^{\varepsilon}:=L^{2}(\Omega)$ (resp. $\left.H^{1}(\Omega)\right)$. Let $|\cdot|_{\varepsilon}$ (resp. $\|\cdot\|_{\varepsilon}$ ) be the Euclidean norm on $L^{2}(\Omega)$ (resp. $H^{1}(\Omega)$ ) induced by $\langle\cdot, \cdot\rangle_{\varepsilon}\left(\right.$ resp. $\left\langle\langle\cdot, \cdot\rangle_{\varepsilon}\right)$. Furthermore, for $u, v \in L_{s}^{2}(\Omega)$ set

$$
\langle u, v\rangle_{0}:=b_{0}(u, v)
$$

Finally, for $u, v \in H_{s}^{1}(\Omega)$ set

$$
\langle\langle u, v\rangle\rangle_{0}:=a_{0}(u, v)+b_{0}(u, v) .
$$

$\langle\cdot, \cdot\rangle_{0}\left(\right.$ resp. $\left.\langle\langle\cdot, \cdot\rangle\rangle_{0}\right)$ is a scalar product on $H^{0}:=L_{s}^{2}(\Omega)\left(\right.$ resp. $\left.H_{s}^{1}(\Omega)\right)$.

Let $|\cdot|_{0}\left(\right.$ resp. $\left.\|\cdot\|_{0}\right)$ be the Euclidean norm on $L_{s}^{2}(\Omega)\left(\right.$ resp. $\left.H_{s}^{1}(\Omega)\right)$ induced by $\langle\cdot, \cdot\rangle_{0}\left(\operatorname{resp} .\langle\langle\cdot, \cdot\rangle\rangle_{0}\right)$.

For $\varepsilon \in[0,1],\left(H^{\varepsilon},\langle\cdot, \cdot\rangle_{\varepsilon}\right)$ is a Hilbert space.

For $\varepsilon \in[0,1]$, the pair $\left(a_{\varepsilon},\langle\cdot, \cdot\rangle_{\varepsilon}\right)$ generates a densely defined selfadjoint operator $\mathbf{A}_{\varepsilon}$ on $\left(H^{\varepsilon},\langle\cdot, \cdot\rangle_{\varepsilon}\right)$.

The (linear) operators $\mathbf{B}_{\varepsilon}$ (resp. $\left.\mathbf{A}_{\varepsilon}\right)$ defined by $\left(\widetilde{a}_{\varepsilon}, \widetilde{b}_{\varepsilon}\right)\left(\operatorname{resp} .\left(a_{\varepsilon}, b_{\varepsilon}\right)\right)$ satisfy the following properties:

(a) $u \in D\left(\mathbf{B}_{\varepsilon}\right)$ if and only if $u \circ\left(\Gamma_{\varepsilon}\right)_{\mid \Omega} \in D\left(\mathbf{A}_{\varepsilon}\right)$;

(b) $\mathbf{A}_{\varepsilon}\left(u \circ\left(\Gamma_{\varepsilon}\right)_{\mid \Omega}\right)=\left(\mathbf{B}_{\varepsilon} u\right) \circ\left(\Gamma_{\varepsilon}\right)_{\mid \Omega}$ for $u \in D\left(\mathbf{B}_{\varepsilon}\right)$.

The following result was proved in [7]

Proposition 3.3. [7, Corollary 4.5] The family $\left(H^{\varepsilon},\langle\cdot, \cdot\rangle_{H^{\varepsilon}}, \mathbf{A}_{\varepsilon}\right)_{\varepsilon \in[0,1]} d e-$ fined in this section satisfies hypothesis (Res).

Now consider the following:

Assumption 3.4. $G:[0,1] \times \mathcal{U} \times \mathbb{R} \rightarrow \mathbb{R},(\varepsilon, y, s) \mapsto G(\varepsilon, y, s)$ is continuous and such that, for all $(\varepsilon, y) \in[0,1] \times \mathcal{U}, G(\varepsilon, y, \cdot)$ is continuously differentiable in s. Moreover, for $\varepsilon \in[0,1], G\left(\varepsilon, \Gamma_{\varepsilon}(\cdot), 0\right)_{\mid \Omega} \in L^{2}(\Omega)$ and $G\left(\varepsilon, \Gamma_{\varepsilon}(\cdot), 0\right)_{\mid \Omega} \rightarrow$ $G(0, \phi(\cdot), 0)_{\mid \Omega}$ in $L^{2}(\Omega)$ as $\varepsilon \rightarrow 0^{+}$. Furthermore, there is a constant $C_{G} \in[0, \infty[$ such that

$$
\left|\partial_{s} G\left(\varepsilon, \Gamma_{\varepsilon}(x), s\right)\right| \leq C_{G}\left(1+|s|^{\beta}\right)
$$

for all $(\varepsilon, x, s) \in[0,1] \times \Omega \times \mathbb{R}$, where $\beta \in] 0, \infty[$ is arbitrary for $\ell=2$ and $\beta=2 /(\ell-2)$ for $\ell \geq 3$.

Finally, the function $G(0, \cdot, \cdot)$ is continuously differentiable in $(y, s)$.

Proposition 3.5. Given $\varepsilon \in[0,1]$ and $u \in H^{1}(\Omega)$ define the function $f_{\varepsilon}(u): \Omega \rightarrow \mathbb{R}$ by

$$
f_{\varepsilon}(u)(x)=G\left(\varepsilon, \Gamma_{\varepsilon}(x), u(x)\right), \quad x \in \Omega .
$$


For all $\varepsilon \in[0,1]$ the function $f_{\varepsilon}(u)$ lies in $H^{\varepsilon}$ for $u \in H_{1}^{\varepsilon}$ and the induced family $\left(f_{\varepsilon}\right)_{\varepsilon \in[0,1]}$ satisfies condition (Conv).

Proof. Since there are continuous imbeddings from $H^{1}(\Omega)$ to $L^{2}(\Omega)$ and from $H^{1}(\Omega)$ to $L^{2(\beta+1)}(\Omega)$, it follows that there is a constant $\left.C_{1} \in\right] 0, \infty[$ such that for all $\varepsilon \in[0,1]$ and all $u \in H^{1}(\Omega), f_{\varepsilon}(u)$ lies in $L^{2}(\Omega)$ and

$$
\left|f_{\varepsilon}(u)\right|_{L^{2}(\Omega)} \leq\left|G\left(\varepsilon, \Gamma_{\varepsilon}(\cdot), 0\right)\right|_{L^{2}(\Omega)}+C_{1}|u|_{H^{1}(\Omega)}^{\beta+1},
$$

and for all all $u, v \in H^{1}(\Omega)$

$$
\left|f_{\varepsilon}(u)-f_{\varepsilon}(v)\right|_{L^{2}(\Omega)} \leq C_{1}\left(|u|_{H^{1}(\Omega)}^{\beta}+|v|_{H^{1}(\Omega)}^{\beta}\right)|u-v|_{H^{1}(\Omega)} .
$$

It follows that, for $\varepsilon \in] 0,1]$, formula (3.5) defines an operator $f_{\varepsilon}: H_{1}^{\varepsilon} \rightarrow H^{\varepsilon}$. We will now show that $f_{0}(u) \in H^{0}$ if $u \in H_{1}^{0}$. To prove this let $\left(\mathcal{U}_{k}\right)_{k \in \mathbb{N}}$ be a covering of $\mathcal{U}$ by a sequence of open sets with $\mathrm{Cl}_{k} \subset \mathcal{U}_{k+1}$ and $\mathrm{Cl} \mathcal{U}_{k}$ compact for all $k \in$ $\mathbb{N}$. For $k \in \mathbb{N}$ let $\xi_{k}: \mathcal{U} \times \mathbb{R} \rightarrow \mathbb{R}$ be a $C^{\infty}$-function with $0 \leq \xi_{k} \leq 1, \xi_{k}(y, t)=1$ for $(y, t) \in S_{k}:=\mathrm{Cl}_{\mathcal{U}} \times[-k, k]$ and $\xi_{k}(y, t)=0$ for $\left.(y, t) \notin \mathcal{U}_{k+1} \times\right]-k-1, k+1[$. Since $u \in H^{1}(\Omega)$, there is a sequence $\left(u_{n}\right)_{n \in \mathbb{N}}$ in $H^{1}(\Omega) \cap C^{\infty}(\Omega)$ with $u_{n} \rightarrow u$ in $H^{1}(\Omega)$. We may assume that $u_{n} \rightarrow u$ and $\partial_{j} u_{n} \rightarrow \partial_{j} u$ almost everywhere in $\Omega$ for all $j \in[1 . . \ell]$. Moreover, we may also assume that there is a $v \in L^{2}(\Omega)$ such that $\left|u_{n}\right| \leq v$ and $\left|\partial_{j} u_{n}\right| \leq v$ almost everywhere on $\Omega$ for all $j \in[1 . . \ell]$. Let $k \in \mathbb{N}$ be arbitrary. For $n \in \mathbb{N}$ let $w_{k, n}: \Omega \rightarrow \mathbb{R}$ be defined by

$$
w_{k, n}(x)=\xi_{k}\left(\phi(x), u_{n}(x)\right) \cdot G\left(0, \phi(x), u_{n}(x)\right), \quad x \in \Omega
$$

It follows that $w_{k, n} \rightarrow w_{k}$ almost everywhere in $\Omega$ as $n \rightarrow \infty$, where $w_{k}: \Omega \rightarrow \mathbb{R}$ is defined by

$$
w_{k}(x)=\xi_{k}(\phi(x), u(x)) \cdot G(0, \phi(x), u(x)), \quad x \in \Omega
$$

Moreover, for $x \in \Omega$ we obtain

$$
\begin{aligned}
\left|w_{k, n}(x)\right| & =\left|\xi_{k}\left(\phi(x), u_{n}(x)\right) \cdot G\left(0, \phi(x), u_{n}(x)\right)\right| \\
& \leq\left|\xi_{k}\left(\phi(x), u_{n}(x)\right)\right| \sup _{(y, s) \in S_{k+1}}|G(0, y, s)|
\end{aligned}
$$

as $\xi_{k}\left(\phi(x), u_{n}(x)\right) \cdot G\left(0, \phi(x), u_{n}(x)\right)=0$ if $\left(\phi(x), u_{n}(x)\right) \notin S_{k+1}$.

The function $w_{k, n}$ lies in $C^{1}(\Omega)$ and using the product rule, we obtain for $x \in \Omega$ and $j \in[1 . . \ell]$

$$
\begin{aligned}
\partial_{j} w_{k, n}(x)= & \xi_{k}\left(\phi(x), u_{n}(x)\right) D_{y} G\left(0, \phi(x), u_{n}(x)\right) \partial_{j} \phi(x) \\
& +\xi_{k}\left(\phi(x), u_{n}(x)\right) \partial_{s} G\left(0, \phi(x), u_{n}(x)\right) \partial_{j} u_{n}(x) \\
& +D_{y} \xi_{k}\left(\phi(x), u_{n}(x)\right) \partial_{j} \phi(x) G\left(0, \phi(x), u_{n}(x)\right) \\
& +\partial_{s} \xi_{k}\left(\phi(x), u_{n}(x)\right) \partial_{j} u_{n}(x) G\left(0, \phi(x), u_{n}(x)\right) .
\end{aligned}
$$


Thus $\partial_{j} w_{k, n} \rightarrow w_{k}^{(j)}$ almost everywhere in $\Omega$ as $n \rightarrow \infty$, where $w_{k}^{(j)}: \Omega \rightarrow \mathbb{R}$ is defined by

$$
\begin{aligned}
w_{k}^{(j)}(x)= & \xi_{k}(\phi(x), u(x)) D_{y} G(0, \phi(x), u(x)) \partial_{j} \phi(x) \\
& +\xi_{k}(\phi(x), u(x)) \partial_{s} G(0, \phi(x), u(x)) \partial_{j} u(x) \\
& +D_{y} \xi_{k}(\phi(x), u(x)) \partial_{j} \phi(x) G(0, \phi(x), u(x)) \\
& +\partial_{s} \xi_{k}(\phi(x), u(x)) \partial_{j} u(x) G(0, \phi(x), u(x)) .
\end{aligned}
$$

Moreover, for $x \in \Omega$,

$$
\begin{aligned}
\left|\partial_{j} w_{k, n}(x)\right| \leq & \left|\xi_{k}\left(\phi(x), u_{n}(x)\right)\right| \sup _{(y, s) \in S_{k+1}}\left|D_{y} G(0, y, s)\right| \sup _{y \in \mathcal{U}}\left|\partial_{j} \phi(y)\right| \\
& +\sup _{(y, s) \in \mathcal{U} \times \mathbb{R}}\left|\xi_{k}(y, s)\right| \sup _{(y, s) \in S_{k+1}}\left|\partial_{s} G(0, y, s)\right| \cdot\left|\partial_{j} u_{n}(x)\right| \\
& +\left|D_{y} \xi_{k}\left(\phi(x), u_{n}(x)\right)\right| \sup _{y \in \mathcal{U}}\left|\partial_{j} \phi(y)\right| \sup _{(y, s) \in S_{k+1}}|G(0, y, s)| \\
& +\sup _{(y, s) \in \mathcal{U} \times \mathbb{R}}\left|\partial_{s} \xi_{k}(y, s)\right| \cdot\left|\partial_{j} u_{n}(x)\right| \sup _{(y, s) \in S_{k+1}}|G(0, y, s)| .
\end{aligned}
$$

Now by the mean-value theorem we have, for all $(x, s) \in \Omega \times \mathbb{R}$ and all $j \in[1 . . \ell]$,

$$
\begin{aligned}
\left|\xi_{k}(\phi(x), s)\right| & \leq\left|\xi_{k}(\phi(x), 0)\right|+\sup _{\theta \in[0,1]}\left|\partial_{s} \xi_{k}(\phi(x), \theta s)\right| \cdot|s|, \\
\left|D_{y} \xi_{k}(\phi(x), s)\right| & \leq\left|D_{y} \xi_{k}(\phi(x), 0)\right|+\sup _{\theta \in[0,1]}\left|\partial_{s} D_{y} \xi_{k}(\phi(x), \theta s)\right| \cdot|s| .
\end{aligned}
$$

Note that $\sup _{\in \mathcal{U}}|D \phi(y)|<\infty$ by [7, Lemma 4.4]. Consequently, there is a constant $\left.C_{2}(k) \in\right] 0, \infty[$ such that for all $n \in \mathbb{N}$, all $x \in \Omega$ and all $j \in[1 . . \ell]$,

$$
\left|w_{k, n}(x)\right| \leq\left(\left|\xi_{k}(\phi(x), 0)\right|+C_{2}(k)\left|u_{n}(x)\right|\right) C_{2}(k)
$$

and

$$
\begin{aligned}
\left|\partial_{j} w_{k, n}(x)\right| \leq & \left(\left|\xi_{k}(\phi(x), 0)\right|+C_{2}(k)\left|u_{n}(x)\right|\right) C_{2}(k) \\
& +C_{2}(k)\left|\partial_{j} u_{n}(x)\right| \\
& +\left(\left|D_{y} \xi_{k}(\phi(x), 0)\right|+C_{2}(k)\left|u_{n}(x)\right|\right) C_{2}(k) \\
& +C_{2}(k)\left|\partial_{j} u_{n}(x)\right| .
\end{aligned}
$$

By our choice of $M$ and $q_{0}$ (see Proposition 3.2) we see that the functions $\left|\xi_{k}(\phi(\cdot), 0)\right|$ and $\left|D_{y} \xi_{k}(\phi(\cdot), 0)\right|$ lie in $L^{2}(\Omega)$, since they are continuous hence measurable, bounded and their supports are subsets of the closed $q_{0} / M$-neighbourhood of $\mathrm{Cl} \mathcal{U}_{k+1}$.

By the dominated convergence theorem we now obtain that $w_{k, n} \rightarrow w_{k}$ and for all $j \in[1 . . \ell], \partial_{j} w_{k, n} \rightarrow w_{k}^{(j)}$ in $L^{2}(\Omega)$ as $n \rightarrow \infty$. Thus $w_{k} \in H^{1}(\Omega)$ and $\partial_{j} w_{k}=w_{k}^{(j)}$ for all $j \in[1 \ldots \ell]$. 
Now, for all $x \in \mathcal{U}$ and all $\nu \in T_{\phi(x)}^{\perp}(\mathcal{M})$,

$$
\sum_{j=1}^{\ell} \partial_{j} \phi(x) \nu_{j}=D \phi(x) \cdot \nu=0 .
$$

Since $u \in H_{s}^{1}(\Omega)$, we infer from (3.4) that $\sum_{j=1}^{\ell} \partial_{j} u(x) \nu_{j}=0$ for almost all $x \in \mathcal{U}$ and all $\nu \in T_{\phi(x)}^{\perp}(\mathcal{M})$. Consequently (3.11) implies that for almost all $x \in \mathcal{U}$ and all $\nu \in T_{\phi(x)}^{\perp}(\mathcal{M}), \sum_{j=1}^{\ell} \partial_{j} w_{k}(x) \nu_{j}=0$. Thus $w_{k} \in H_{s}^{1}(\Omega)$ for all $k \in \mathbb{N}$. Now $w_{k} \rightarrow G(0, \phi(\cdot), u(\cdot))$ almost everywhere in $\Omega$ as $k \rightarrow \infty$. Moreover, $\left|w_{k}\right| \leq$ $|G(0, \phi(\cdot), u(\cdot))|$ almost everywhere in $\Omega$. Since $G(0, \phi(\cdot), u(\cdot)) \in L^{2}(\Omega)$, it follows that $w_{k} \rightarrow G(0, \phi(\cdot), u(\cdot))$ in $L^{2}(\Omega)$ as $k \rightarrow \infty$. Hence $G(0, \phi(\cdot), u(\cdot)) \in$ $L_{s}^{2}(\Omega)$. Our claim is proved. Thus part (a) of condition (Conv) holds with $\bar{\varepsilon}=1$.

Now (3.6) together with [7, Propositions 2.7 and 2.8] imply that part (c) of condition (Conv) holds. Our assumptions together with dominated convergence theorem imply that whenever $u \in H_{s}^{1}(\Omega)$, then $f_{\varepsilon}(u) \rightarrow f_{0}(u)$ in $L^{2}(\Omega)$ as $\varepsilon \rightarrow 0$. This together with Theorem 2.4 and [7, Proposition 2.7] shows that the remaining parts (b) and (d) of condition (Conv) hold.

We will now prove that under some additional hypotheses on $\left(f_{\varepsilon}\right)_{\varepsilon \in[0,1]}$, whenever $\beta \in] 0, \infty\left[\right.$ and $N$ is a closed bounded subset of $H_{1}^{0}$, then $N$ is strongly admissible rel. to $\beta$ and the family $\left(H^{\varepsilon}, \pi_{\varepsilon}\right)_{\varepsilon \in[0, \bar{\varepsilon}]}$. Here, for $\varepsilon \in[0,1], \pi_{\varepsilon}$ is the local semiflow generated on $H_{\varepsilon}^{1}$ by the semilinear differential equation

$$
\dot{u}=-\mathbf{A}_{\varepsilon} u+f_{\varepsilon}(u) .
$$

Our guiding principle is the method of tail estimates, cf. [18], [9], [1], [12], which we adapt to the present situation.

Define the function $\lambda: \Omega \rightarrow \mathbb{R}$ by

$$
\lambda(x)=\langle\phi(x), \phi(x)\rangle, \quad x \in \Omega .
$$

Moreover, let $\vartheta: \mathbb{R} \rightarrow \mathbb{R}$ be a $C^{\infty}$-function such that $0 \leq \vartheta \leq 1, \vartheta=0$ on $\left.]-\infty, 1\right]$ and $\vartheta=1$ on $\left[2, \infty\left[\right.\right.$. For $k \in \mathbb{N}$ define the function $\theta_{k}: \Omega \rightarrow \mathbb{R}$ by

$$
\theta_{k}(x)=\vartheta\left(\lambda(x) / k^{2}\right), \quad x \in \Omega .
$$

Then $\theta_{k}$ is a $C^{1}$-function and for all $x \in \Omega$ and all $\nu \in \mathbb{R}^{\ell}$,

$$
\left\langle\nabla \theta_{k}(x), \nu\right\rangle=D \theta_{k}(x) . \nu=\left(2 / k^{2}\right) \vartheta^{\prime}\left(\lambda(x) / k^{2}\right)\langle D \phi(x) . \nu, \phi(x)\rangle .
$$

In particular,

$$
\left\langle\nabla \theta_{k}(x), \nu\right\rangle=0, \quad x \in \Omega, \nu \in T_{\phi(x)}^{\perp}(\mathcal{M}) .
$$


Now, if $u \in H^{1}(\Omega)$, then $\theta_{k} \cdot u \in H^{1}(\Omega)$. For almost all $x \in \Omega, \nu_{x}=P(x) \nabla u(x) \in$ $T_{\phi(x)}^{\perp}(\mathcal{M})$ so

$$
\left\langle P(x) \nabla \theta_{k}(x), P(x) \nabla u(x)\right\rangle=0 .
$$

Now let $\varepsilon \in[0,1]$ and $u \in H_{1}^{\varepsilon}$ be arbitrary. If $\varepsilon>0$, then we obtain from (3.16)

$$
\begin{aligned}
-a_{\varepsilon}\left(\theta_{k} \cdot u, u\right)= & -\int_{\Omega} J_{\varepsilon}(x) \theta_{k}(x)\left\langle S_{\varepsilon}(x)^{T} \nabla u(x), S_{\varepsilon}(x)^{T} \nabla u(x)\right\rangle d x \\
& -\int_{\Omega} J_{\varepsilon}(x) u(x)\left\langle S_{\varepsilon}(x)^{T} \nabla \theta_{k}(x), S_{\varepsilon}(x)^{T} \nabla u(x)\right\rangle d x \\
& -\frac{1}{\varepsilon^{2}} \int_{\Omega} J_{\varepsilon}(x) \theta_{k}\langle P(x) \nabla u(x), P(x) \nabla u(x)\rangle d x \\
& -\frac{1}{\varepsilon^{2}} \int_{\Omega} J_{\varepsilon}(x) u(x)\left\langle P(x) \nabla \theta_{k}(x), P(x) \nabla u(x)\right\rangle d x \\
\leq & -\int_{\Omega} J_{\varepsilon}(x) u(x)\left\langle S_{\varepsilon}(x)^{T} \nabla \theta_{k}(x), S_{\varepsilon}(x)^{T} \nabla u(x)\right\rangle d x .
\end{aligned}
$$

If $\varepsilon=0$ and $u \in H_{s}^{1}(\Omega)$ then, by (3.4) and (3.15), $\theta_{k} \cdot u \in H_{s}^{1}(\Omega)$ and we obtain

$$
\begin{aligned}
-a_{\varepsilon}\left(\theta_{k} \cdot u, u\right)= & -\int_{\Omega} J_{\varepsilon}(x) \theta_{k}(x)\left\langle S_{\varepsilon}(x)^{T} \nabla u(x), S_{\varepsilon}(x)^{T} \nabla u(x)\right\rangle d x \\
& -\int_{\Omega} J_{\varepsilon}(x) u(x)\left\langle S_{\varepsilon}(x)^{T} \nabla \theta_{k}(x), S_{\varepsilon}(x)^{T} \nabla u(x)\right\rangle d x \\
\leq & -\int_{\Omega} J_{\varepsilon}(x) u(x)\left\langle S_{\varepsilon}(x)^{T} \nabla \theta_{k}(x), S_{\varepsilon}(x)^{T} \nabla u(x)\right\rangle d x .
\end{aligned}
$$

Thus, whenever $\varepsilon \in[0,1]$ and $u \in H_{1}^{\varepsilon}$ then $\theta_{k} \cdot u \in H_{1}^{\varepsilon}$ and

$$
-a_{\varepsilon}\left(\theta_{k} \cdot u, u\right) \leq-\int_{\Omega} J_{\varepsilon}(x) u(x)\left\langle S_{\varepsilon}(x)^{T} \nabla \theta_{k}(x), S_{\varepsilon}(x)^{T} \nabla u(x)\right\rangle d x .
$$

Now, setting $h(x)=S(x) S^{T}(x) \nabla u(x)$ we see that

$$
\begin{aligned}
\left\langle S_{\varepsilon}(x)^{T} \nabla \theta_{k}(x), S_{\varepsilon}(x)^{T} \nabla u(x)\right\rangle & =D \theta_{k}(x) \cdot h(x) \\
& =\left(2 / k^{2}\right) \vartheta^{\prime}\left(\lambda(x) / k^{2}\right)\langle\phi(x), D \phi(x) . h(x)\rangle .
\end{aligned}
$$

Thus, using (3.17) and letting $\Omega_{k}$ be the set of all $x \in \Omega$ with $k^{2} \leq \lambda(x) \leq 2 k^{2}$ we see that

$$
-a_{\varepsilon}\left(\theta_{k} \cdot u, u\right) \leq\left(2 / k^{2}\right) C^{\prime} \int_{\Omega_{k}}|u(x)| \sup _{y \in \Omega_{k}}|\phi(y)||\nabla u(x)| d x
$$

where

$$
C^{\prime}=\sup _{\varepsilon \in[0,1], y \in \mathcal{U}} J_{\varepsilon}(y) \sup _{s \in \mathbb{R}}\left|\vartheta^{\prime}(s)\right| \sup _{y \in \mathcal{U}}|D \phi(y)| \sup _{\varepsilon \in[0,1], y \in \mathcal{U}}\left|S_{\varepsilon}(y) S_{\varepsilon}^{T}(y)\right| .
$$

Our choice of $\vartheta$ and [7, Lemma 4.4] imply that $C^{\prime}<\infty$. Thus

$$
-a_{\varepsilon}\left(\theta_{k} \cdot u, u\right) \leq\left(C_{1} / k\right) \int_{\Omega}|u(x)||\nabla u(x)| d x \leq\left(C_{11} / k\right)|u|_{H^{1}(\Omega)}^{2} .
$$


Here, $C_{1}=2 \sqrt{2} C^{\prime}$. We thus obtain

Lemma 3.6. There is a constant $\left.C_{1} \in\right] 0, \infty[$ such that for all $k \in \mathbb{N}$, all $\varepsilon \in[0,1]$ and all $u \in H_{1}^{\varepsilon}$ we have $\theta_{k} \cdot u \in H_{1}^{\varepsilon}$ and

$$
-a_{\varepsilon}\left(\theta_{k} \cdot u, u\right) \leq\left(C_{1} / k\right)|u|_{H^{1}(\Omega)}^{2} .
$$

Lemma 3.7. Let $\varepsilon \in[0,1], k \in \mathbb{N}$ and $T \in] 0, \infty[$ be arbitrary and $u:[0, T] \rightarrow$ $H_{1}^{\varepsilon}$ be a solution of

$$
\dot{u}=-\mathbf{A}_{\varepsilon} u+f_{\varepsilon}(u) .
$$

Then the function $\zeta:[0, T] \rightarrow \mathbb{R}, t \mapsto b_{\varepsilon}\left(\theta_{k} \cdot u(t), u(t)\right)$ is continuously differentiable on $[0, T]$ and

$$
\zeta^{\prime}(t)=\xi(t):=-2 a_{\varepsilon}\left(\theta_{k} \cdot u(t), u(t)\right)+2 b_{\varepsilon}\left(\theta_{k} \cdot u(t), f_{\varepsilon}(u(t))\right), \quad t \in[0, T] .
$$

For every $\mu \in] 0, \infty[$

$$
\zeta(t)=e^{-2 \mu t} \zeta(0)+\int_{0}^{t} e^{-2 \mu(t-s)} \xi_{\mu}(s) d s, \quad t \in[0, T],
$$

where $\xi_{\mu}:[0, T] \rightarrow \mathbb{R}$ is defined by

$$
\xi_{\mu}(t)=-2 a_{\varepsilon}\left(\theta_{k} \cdot u(t), u(t)\right)+2 b_{\varepsilon}\left(\theta_{k} \cdot u(t), f_{\varepsilon}(u(t))+\mu u(t)\right), \quad t \in[0, T] .
$$

If $R \in\left[0, \infty\left[\right.\right.$ is such that $|u(t)|_{H^{1}(\Omega)} \leq R$ for all $t \in[0, T]$ then

$$
\left|\theta_{k} \cdot u(t)\right|_{L^{2}(\Omega)}^{2} \leq C_{3}^{-1}\left(e^{-2 \mu t} C_{2} R^{2}+\left(C_{1} R^{2} /(\mu k)\right)+\left(M_{k}(R) / \mu\right)\right)
$$

where $C_{2}=\sup _{\varepsilon \in[0,1]} \sup _{x \in \mathcal{U}} J_{\varepsilon}(x)<\infty, C_{3}=\inf _{\varepsilon \in[0,1]} \sup _{x \in \mathcal{U}} J_{\varepsilon}(x)>0$ and

$$
M_{k}(R)=\sup _{\varepsilon \in[0,1], u \in H_{1}^{\varepsilon},|u|_{H^{1}(\Omega)} \leq R} b_{\varepsilon}\left(\theta_{k} \cdot u, f_{\varepsilon}(u)+\mu u\right) .
$$

Proof. Since the map $H^{1}(\Omega) \rightarrow H^{1}(\Omega), v \mapsto \theta_{k} \cdot v$ is linear and bounded and since the norm $|\cdot|_{H_{1}^{\varepsilon}}$ is equivalent (with bounds depending on $\varepsilon$ ) to the (restriction of the) norm of $H^{1}(\Omega)$, it follows that the map $H_{1}^{\varepsilon} \rightarrow H_{1}^{\varepsilon}, v \mapsto \theta_{k} \cdot v$ is well-defined, linear and bounded. Since $u$ is continuous (into $H_{1}^{\varepsilon}$ ) and the restriction of $u$ to $] 0, T$ ] is differentiable into $H^{\varepsilon}$, it follows that $\zeta$ and $\xi$ are continuous on $[0, T]$. Moreover, $\zeta$ differentiable on $] 0, T]$ and, for $t \in] 0, T]$,

$$
\begin{aligned}
\zeta^{\prime}(t) & =b_{\varepsilon}\left(\theta_{k} \cdot u^{\prime}(t), u(t)\right)+b_{\varepsilon}\left(\theta_{k} \cdot u(t), u^{\prime}(t)\right) \\
& =2 b_{\varepsilon}\left(\theta_{k} \cdot u(t), u^{\prime}(t)\right)=2 b_{\varepsilon}\left(\theta_{\varepsilon} \cdot u(t),-\mathbf{A}_{\varepsilon} u(t)+f_{\varepsilon}(u(t))\right) .
\end{aligned}
$$

Since, for $w \in H_{1}^{\varepsilon}$ and $v \in D\left(\mathbf{A}_{\varepsilon}\right)$,

$$
b_{\varepsilon}\left(w, \mathbf{A}_{\varepsilon} v\right)=a_{\varepsilon}(w, v),
$$

it follows that $\zeta$ is differentiable on $] 0, T]$ and $\zeta^{\prime}(t)=\xi(t)$ for $\left.\left.t \in\right] 0, T\right]$. Since $\xi$ is continuous at $t=0$ it follows that $\zeta$ is differentiable at $t=0$ and $\zeta^{\prime}(0)=\xi(0)$. 
We now conclude that, given $\mu \in] 0, \infty[$

$$
\zeta^{\prime}(t)=-2 \mu \zeta(t)+\xi_{\mu}(t), \quad t \in[0, T] .
$$

Now the variation-of-constants formula proves (3.18).

To prove (3.19), note, that formula (3.18), Lemma 3.6 and the fact that $0 \leq \theta_{k} \leq 1$ imply

$$
C_{3}\left|\theta_{k} \cdot u(t)\right|_{L^{2}(\Omega)} \leq \zeta(t) \leq e^{-2 \mu t} \zeta(0)+(1 / 2 \mu)\left(2\left(C_{1} / k\right) R^{2}+2 M_{k}(R)\right) .
$$

Since $\zeta(0) \leq C_{2}|u(0)|_{L^{2}(\Omega)}^{2} \leq C_{2} R^{2}$, estimate (3.19) follows.

Let us call the family $\left(f_{\varepsilon}\right)_{\varepsilon \in[0,1]}$ tail admissible if there is a $\left.\mu \in\right] 0, \infty[$ such that for every $k \in \mathbb{N}$ and every $R \in] 0, \infty[$

$$
M_{k}(R)=\sup _{\varepsilon \in[0,1], u \in H_{1}^{\varepsilon},|u|_{H^{1}(\Omega)} \leq R} b_{\varepsilon}\left(\theta_{k} \cdot u, f_{\varepsilon}(u)+\mu u\right) \rightarrow 0, \text { as } k \rightarrow \infty .
$$

The following sufficient conditions for tail admissibility are due to Prizzi [9] in the context of reaction-diffusion equations on $\mathbb{R}^{\ell}$.

Lemma 3.8. Assume there are numbers $\mu \in] 0, \infty[, q \in[2, \infty[$ and $p \in] 1, \infty]$ and functions $c \in L^{1}(\Omega)$, $e \in L^{p}(\Omega)$ such that

(a) $s \cdot G\left(\varepsilon, \Gamma_{\varepsilon}(x), s\right) \leq-\mu|s|^{2}+e(x)|s|^{q}+c(x)$ for all $\varepsilon \in[0,1], s \in \mathbb{R}$ and a.a. $x \in \Omega$.

(b) If $\ell=2$ and $p=\infty$, then $\left|\theta_{k} \cdot e\right|_{L^{\infty}(\Omega)} \rightarrow 0$ as $k \rightarrow \infty$.

(c) For $\ell \geq 3$ either $q<2 \ell /(\ell-2)$ and $2 \ell /(2 \ell-q(\ell-2)) \leq p<\infty$ or else $q \leq 2 \ell /(\ell-2), p=\infty$ and $\left|\theta_{k} \cdot e\right|_{L^{\infty}(\Omega)} \rightarrow 0$ as $k \rightarrow \infty$.

Then $\left(f_{\varepsilon}\right)_{\varepsilon \in[0,1]}$ is tail admissible.

Proof. Let $r=p /(p-1)$ for $p<\infty$ and $r=1$ for $p=\infty$. For $\varepsilon \in[0,1]$, $k \in \mathbb{N}$ and $u \in H_{1}^{\varepsilon}$ we have, by Hölder inequality,

$$
\begin{aligned}
b_{\varepsilon}\left(\theta_{k} \cdot u, f_{\varepsilon}(u)+\mu u\right) & =\int_{\Omega} J_{\varepsilon}(x) \theta_{k}(x) u(x)\left(G\left(\varepsilon, \Gamma_{\varepsilon}(x), u(x)\right)+\mu u(x)\right) d x \\
& \leq \int_{\Omega} J_{\varepsilon}(x) \theta_{k}(x)\left(e(x)|u(x)|^{q}+c(x)\right) d x \\
& \leq C_{2}\left(\left|\theta_{k} \cdot e\right|_{L^{p}(\Omega)}|u|_{L^{q r}(\Omega)}^{q}+\left|\theta_{k} \cdot c\right|_{L^{1}(\Omega)}\right)
\end{aligned}
$$

where, as before, $C_{2}=\sup _{\varepsilon \in[0,1]} \sup _{x \in \mathcal{U}} J_{\varepsilon}(x)<\infty$. By our assumptions there is a bounded imbedding from $H^{1}(\Omega)$ to $L^{q r}(\Omega)$. Thus there is a constant $C_{4} \in$ ] $0, \infty\left[\right.$, such that for all $\varepsilon \in[0,1]$, all $k \in \mathbb{N}$ and all $u \in H_{1}^{\varepsilon}$

$$
b_{\varepsilon}\left(\theta_{k} \cdot u, f_{\varepsilon}(u)+\mu u\right) \leq C_{2}\left(C_{4}\left|\theta_{k} \cdot e\right|_{L^{p}(\Omega)}|u|_{H^{1}(\Omega)}^{q}+\left|\theta_{k} \cdot c\right|_{L^{1}(\Omega)}\right) .
$$

By the properties of $\vartheta$ and the dominated convergence theorem we obtain that $\left|\theta_{k} \cdot c\right|_{L^{1}(\Omega)} \rightarrow 0$ and $\left|\theta_{k} \cdot e\right|_{L^{p}(\Omega)} \rightarrow 0$ (for $p<\infty$ ) for $k \rightarrow \infty$. But by our 
assumption, the latter convergence also holds for $p=\infty$. Together with (3.20), this implies the lemma.

We can now state the following

Theorem 3.9. If $\left(f_{\varepsilon}\right)_{\varepsilon \in[0,1]}$ is tail admissible, $\left.\beta \in\right] 0, \infty[$ and $N$ is a closed bounded subset of $H_{1}^{0}$, then $N$ is strongly admissible rel. to $\beta$ and the family $\left(H^{\varepsilon}, \pi_{\varepsilon}\right)_{\varepsilon \in[0, \bar{\varepsilon}]}$.

Proof. Since for each $\varepsilon \in[0,1]$ the map $f_{\varepsilon}$ maps bounded subsets of $H_{1}^{\varepsilon}$ to bounded subsets of $H^{\varepsilon}$, it follows that $\pi_{\varepsilon}$ does not explode in $N_{\varepsilon, \beta}$. There is a bound $R \in] 0, \infty\left[\right.$ such that $|u|_{H^{1}(\Omega)} \leq R$ for all $\varepsilon \in[0,1]$ and $u \in N_{\varepsilon, \beta}$.

Let $\varepsilon_{0} \in[0,1],\left(\varepsilon_{n}\right)_{n}$ be a sequence in $[0, \bar{\varepsilon}],\left(t_{n}\right)_{n}$ be a sequence in $] 0, \infty[$ and $\left(u_{n}\right)_{n}$ be a sequence such that

$$
t_{n} \rightarrow \infty \text { as } n \rightarrow \infty,
$$

either $\varepsilon_{n}=\varepsilon_{0}$ for all $n \in \mathbb{N}$ or else $\varepsilon_{0}=0$ and $\varepsilon_{n} \rightarrow \varepsilon_{0}$ for $n \rightarrow \infty$,

and, for each $n \in \mathbb{N}, u_{n} \in H_{1}^{\varepsilon_{n}}$ and $u_{n} \pi_{\varepsilon_{n}}\left[0, t_{n}\right] \subset N_{\varepsilon_{n}, \beta}$.

If $\varepsilon_{n}=\varepsilon_{0}$ for all $n \in \mathbb{N}$, then $\left(u_{n} \pi_{\varepsilon_{0}} t_{n}\right)_{n}$ is bounded in the Hilbert space $H_{1}^{\varepsilon_{0}}$, so there is a $v \in H_{1}^{\varepsilon_{0}} \subset H^{1}(\Omega)$ and a subsequence of $\left(u_{n} \pi_{\varepsilon_{0}} t_{n}\right)_{n}$, again denoted by $\left(u_{n} \pi_{\varepsilon_{0}} t_{n}\right)_{n}$, such that $\left(u_{n} \pi_{\varepsilon_{0}} t_{n}\right)_{n}$ converges weakly in $H_{1}^{\varepsilon_{0}}$, hence in $H^{1}(\Omega)$ to $v$. If $\varepsilon_{0}=0$ and $\varepsilon_{n} \rightarrow \varepsilon_{0}$ for $n \rightarrow \infty$, then $\left(u_{n} \pi_{\varepsilon_{n}} t_{n}\right)_{n}$ is bounded in the Hilbert space $H^{1}(\Omega)$, so there is a $v \in H^{1}(\Omega)$ and a subsequence of $\left(u_{n} \pi_{\varepsilon_{n}} t_{n}\right)_{n}$, again denoted by $\left(u_{n} \pi_{\varepsilon_{n}} t_{n}\right)_{n}$, such that $\left(u_{n} \pi_{\varepsilon_{n}} t_{n}\right)_{n}$ converges weakly in $H^{1}(\Omega)$ to $v$. Thus $\left(P(, \cdot) \nabla\left(u_{n} \pi_{\varepsilon_{n}} t_{n}\right)\right)_{n}$ converges weakly in $L^{2}\left(\Omega, \mathbb{R}^{\ell}\right)$ to $P(\cdot) \nabla v$. From the definition of $a_{\varepsilon}$ it follows that $\left|P(\cdot) \nabla\left(u_{n} \pi_{\varepsilon_{n}} t_{n}\right)\right|_{L^{2}\left(\Omega, \mathbb{R}^{\ell}\right)} \rightarrow 0$. Thus $\left(P(\cdot) \nabla\left(u_{n} \pi_{\varepsilon_{n}} t_{n}\right)\right)_{n}$ converges to 0 strongly hence weakly in $L^{2}\left(\Omega, \mathbb{R}^{\ell}\right)$. This shows that $P(\cdot) \nabla v=0$ almost everywhere in $\Omega$, i.e. $v \in H_{s}^{1}(\Omega)=H_{1}^{\varepsilon_{0}}$.

The proof will be completed if we can show that there is a subsequence of $\left(u_{n} \pi_{\varepsilon_{n}} t_{n}\right)_{n}$, again denoted by $\left(u_{n} \pi_{\varepsilon_{n}} t_{n}\right)_{n}$, such that $\left|u_{n} \pi_{\varepsilon_{n}} t_{n}-v\right|_{H^{\varepsilon_{n}}} \rightarrow 0$ as $n \rightarrow \infty$, i.e. equivalently, that $\left|u_{n} \pi_{\varepsilon_{n}} t_{n}-v\right|_{L^{2}(\Omega)} \rightarrow 0$ as $n \rightarrow \infty$. By the uniqueness of weak limits we thus have to show that the sequence $\left(u_{n} \pi_{\varepsilon_{n}} t_{n}\right)_{n}$ is precompact in $L^{2}(\Omega)$, i.e. that $\alpha\left(\left\{u_{n} \pi_{\varepsilon_{n}} t_{n} \mid n \in \mathbb{N}\right\}\right)=0$, where $\alpha$ is the Kuratowski measure of noncompactness on $L^{2}(\Omega)$. We have for every $k \in \mathbb{N}$

$$
\begin{aligned}
\alpha\left(\left\{u_{n} \pi_{\varepsilon_{n}} t_{n} \mid n \in \mathbb{N}\right\}\right) \leq & \alpha\left(\left\{\theta_{k} \cdot\left(u_{n} \pi_{\varepsilon_{n}} t_{n}\right) \mid n \in \mathbb{N}\right\}\right) \\
& +\alpha\left(\left\{\left(1-\theta_{k}\right) \cdot\left(u_{n} \pi_{\varepsilon_{n}} t_{n}\right) \mid n \in \mathbb{N}\right\}\right) .
\end{aligned}
$$

Let $\delta \in] 0, \infty[$ be arbitrary. Then (3.19) together with tail admissibility imply that there is a $k \in \mathbb{N}$ and an $n_{0} \in \mathbb{N}$ such that $\left|\theta_{k} \cdot\left(u_{n} \pi_{\varepsilon_{n}} t_{n}\right)\right|_{L^{2}(\Omega)}<\delta$ for all $n \geq n_{0}$. Thus

$$
\alpha\left(\left\{\theta_{k} \cdot\left(u_{n} \pi_{\varepsilon_{n}} t_{n}\right) \mid n \in \mathbb{N}\right\} \leq 2 \delta\right.
$$


Now define $V_{k}$ to be the set of all $x \in \Omega$ with $\|x\|<\left(q_{0} / M\right)+k \sqrt{2}$. Let $S=\left\{\left(1-\theta_{k}\right) \cdot\left(u_{n} \pi_{\varepsilon_{n}} t_{n}\right) \mid n \in \mathbb{N}\right\}$ and $\left(w_{m}\right)_{m}$ be any sequence in $S$. For each $m \in \mathbb{N}$ let $\widetilde{w}_{m}$ be the restriction of $w_{m}$ to the open set $V_{k}$. Then $\widetilde{w}_{m} \in H^{1}\left(V_{k}\right)$ and $\left|\widetilde{w}_{m}\right|_{H^{1}\left(V_{k}\right)} \leq R$. Since $V_{k}$ is bounded, Rellich theorem implies that there is a subsequence $\left(\widetilde{w}_{m_{\ell}}\right)_{\ell}$ of $\left(\widetilde{w}_{m}\right)_{m}$ converging in $L^{2}\left(V_{k}\right)$ to some $\widetilde{w} \in L^{2}\left(V_{k}\right)$. Our choice of $\vartheta$ implies that $w_{m}(x)=0$, whenever $m \in \mathbb{N}, x \in \Omega$ and $x \notin V_{k}$. Thus $\left(w_{m_{\ell}}\right)_{\ell}$ converges in $L^{2}(\Omega)$ to the function $w: \Omega \rightarrow \mathbb{R}$ defined by $w(x)=\widetilde{w}(x)$ if $x \in V_{k}$ and $w(x)=0$ otherwise. Altogether we see that every sequence in $S$ has a subsequence which converges in $L^{2}(\Omega)$. Thus $\alpha(S)=0$ and so $\alpha\left(\left\{u_{n} \pi_{\varepsilon_{n}} t_{n} \mid n \in \mathbb{N}\right\}\right) \leq 2 \delta$. Since $\left.\delta \in\right] 0, \infty[$ is arbitrary, it follows that $\alpha\left(\left\{u_{n} \pi_{\varepsilon_{n}} t_{n} \mid n \in \mathbb{N}\right\}\right)=0$. This completes the proof of the theorem.

We thus obtain the following result.

Theorem 3.10. For $\left(H^{\varepsilon},\langle\cdot, \cdot\rangle_{H^{\varepsilon}}, \mathbf{A}_{\varepsilon}\right)_{\varepsilon \in[0,1]}$ and $\left(f_{\varepsilon}\right)_{\varepsilon \in[0,1]}$ defined in this section, if $\left(f_{\varepsilon}\right)_{\varepsilon \in[0,1]}$ is tail admissible, then the assumptions and hence the conclusions of the Conley index continuation results Theorems 2.17 and 2.18 hold (together with the corresponding remarks).

\section{REFERENCES}

[1] F. Antoci And M. PRIzzi, Reaction-diffusion equations on unbounded thin domains, Topol. Methods Nonlinear Anal. 18 (2001), 283-302.

[2] M.C. Carbinatto and K.P. Rybakowski, Conley index continuation and thin domain problems, Topol. Methods Nonlinear Anal. 16 (2000), 201-251.

[3] , Localized singularities and Conley index, Topol. Methods Nonlinear Anal. 37 (2011), 1-36

[4] On a general Conley index continuation principle for singular perturbation problems, Ergodic Theory Dynam. Systems 22 (2002), 729-755.

[5] - Continuation of the connection matrix in singular perturbation problems, Ergodic Theory Dynam. Systems 26 (2006), 1021-1059.

[6] Continuation of the connection matrix for singularly perturbed hyperbolic equations, Fund. Math. 196 (2007), 253-273.

[7] Resolvent convergence for Laplace operators on unbounded curved squeezed domains, Topol. Methods Nonlinear Anal. 42 (2013), 233-257.

[8] D. Henry, Geometric Theory of Semilinear Parabolic Equations, Lecture Notes in Mathematics, vol. 840, Springer-Verlag, New York, 1981.

[9] M. PRIzzI, On admissibility for parabolic equations in $\mathbb{R}^{n}$, Fund.Math. 176 (2003), $261-275$.

[10] M. PRizzi And K. P. RyBakowski, The effect of domain squeezing upon the dynamics of reaction-diffusion equations, J. Differential Equations 173 (2001), 271-320.

[11] Some recent results on thin domain problems, Topol. Methods Nonlinear Anal. 14 (1999), 239-255.

[12] , Attractors for reaction-diffusion equations on arbitrary unbounded domains, Topol Methods Nonlinear Anal. 30 (2007), 251-270. 
[13] M. Prizzi, M. Rinaldi And K.P. Rybakowski, Curved thin domains and parabolic equations, Studia Math. 151 (2002), 109-140.

[14] K.P. Rybakowski, On the homotopy index for infinite-dimensional semiflows, Trans. Amer. Math. Soc. 269 (1982), 351-382.

[15] , The Homotopy Index and Partial Differential Equations, Springer-Verlag, Berlin, 1987.

[16] - On curved squeezing and Conley index, Topol. Methods Nonlinear Anal. 38 (2011), 207-231.

[17] _ Curved squeezing of unbounded domains and attractors, Journ. Fixed Point Theory and Appl. (to appear).

[18] B. WANG, Attractors for reaction-diffusion equations in unbounded domains, Physica D 128 (1999), 41-52.

Manuscript received January 1, 2014

Krzysztof P. RyBakowski

Universität Rostock, Institut für Mathematik

Ulmenstraße 69, Haus 3

18057 Rostock, GERMANY

E-mail address: krzysztof.rybakowski@uni-rostock.de 